Research Article

\title{
Evaluation of the Therapeutic Effect of Traditional Chinese Medicine on Osteoarthritis: A Systematic Review and Meta-Analysis
}

\author{
Lin Wang $\triangle{ }^{1}$ Xiao-Fei Zhang, ${ }^{1}$ Xue Zhang $\triangle{ }^{1}{ }^{1}$ Dong-Yan Guo, ${ }^{1}$ Yu-Wei Duan, ${ }^{1}$ \\ Zhi-Chao Wang, ${ }^{1}$ Li-Shan Pei, ${ }^{1}$ Han Ru, ${ }^{1}$ Jiang-Xue Cheng, ${ }^{1}$ Ya-Jun Shi $\odot{ }^{1},{ }^{1}$ \\ and Jun-Bo Zou $\mathbb{i D}^{1,2,3}$ \\ ${ }^{1}$ Shaanxi Province Key Laboratory of New Drugs and Chinese Medicine Foundation Research, Pharmacy College,
Shaanxi University of Chinese Medicine, Xianyang 712046, China
${ }^{2}$ Pharmaceutical Factory of Shaanxi University of Chinese Medicine, Xianyang, China
${ }^{3}$ School of Pharmacy, Health Science Center, Xi'an Jiaotong University, Xi'an, China
}

Correspondence should be addressed to Ya-Jun Shi; 2051004@sntcm.edu.cn and Jun-Bo Zou; 2051078@sntcm.edu.cn

Received 23 June 2020; Revised 22 September 2020; Accepted 21 November 2020; Published 14 December 2020

Academic Editor: Jacob Ablin

Copyright (c) 2020 Lin Wang et al. This is an open access article distributed under the Creative Commons Attribution License, which permits unrestricted use, distribution, and reproduction in any medium, provided the original work is properly cited.

Background. Osteoarthritis (OA) is a common degenerative disease of bone and joint characterized by the damage of articular cartilage and hypertonia, which often occurs in the middle-aged and elderly. Traditional Chinese medicine (TCM) therapy, including acupuncture (ACU), oral administration, and external use of traditional Chinese medicines (TCMs), can significantly improve the therapeutic effect on OA and reduce the occurrence of side effects. We provide a latest meta-analysis on the treatment of OA with TCM. Materials and Methods. In the electronic database, appropriate articles without language restrictions on keywords were selected until August 1, 2019. All trajectories are screened according to certain criteria. The quality of qualified research was also assessed. We have made a detailed record of the results of the measurement. Meta-analysis was carried out with Revman 5.3 software. Results. Forty-four articles involving 4014 patients (2012 cases in the experimental group and 2002 cases in the control group) with OA were selected. This article focuses on the study of the treatment of OA by using the general mode of TCM. The quality evaluation included in the study was evaluated independently according to the Cochrane intervention system evaluation manual. In this meta-analysis, $68.18 \%$ of the literature correctly described the conditions for the generation of random assignment sequences, only $6.82 \%$ of the literature correctly mentioned the hidden details of allocation, and all studies mentioned randomly assigned participants. Compared with Western medicine, the total effective rate (TER) of OA treatment in TCM was significantly increased and the recurrence rate $(\mathrm{RR})$ was significantly decreased $(P<0.00001)$. In addition, the experimental group was also superior to the control group in terms of the indicators of joint activity function, inflammatory factor content, and various indicators affecting bone metabolism. It can be showed by the network analysis diagram that Aconiti Radix, Achyranthis Bidentatae Radix, and other TCMs can inhibit inflammatory stimulation and relieve the pain symptoms of patients with OA. ACU at Yinlingquan, Xiyan, and other acupoints can effectively improve the clinical symptoms of patients with OA. Conclusion. TCM therapy in treatment of patients with OA could effectively restore joint function, enhance the TER, and reduce RR. However, the results of this study should be handled with care due to the limitations existing. Some rigorous randomized controlled trials (RCTs) are needed to confirm these findings.

\section{Introduction}

Osteoarthritis (OA) is a chronic degenerative joint disease, which occurs frequently in the elderly, and OA is one of the diseases that lead to disability in the world [1]. The effect of this disease is significant in the knee, hip, and hands [2]. At this stage, the concept of OA has been further developed. OA is considered to be an entire joint disease, including changes in the articular cartilage, subcartilage bone, ligaments, capsule, and synovium, resulting in functional joint injury 
[3], resulting in joint pain and physical disability [4], thus impairing the quality of life of patients with OA.

$\mathrm{OA}$ is considered to be the main cause of chronic pain in clinics. The pain symptoms associated with OA lead to the decrease of physical fitness and behavior ability [5]. In the world today, the trials of pain and disability caused by $\mathrm{OA}$ are interdisciplinary, involving surgical treatment, drugs, and physiotherapy [6]. However, the cost of physiotherapy is important for patients, and the evidence that physiotherapy is effective in treating $\mathrm{OA}$ patients is not clear [7]. Although hip and knee replacement has become a routine treatment for end-stage arthritis, the risk of surgery is high, including the mortality rate caused by surgery and the failure rate of reoperation due to failed implants. Also, the cost of operation also has significant cost effectiveness [8]. The main goal of drug therapy is not only to control joint pain but also to avoid the toxic effects of treatment [9]. Nonsteroidal anti-inflammatory drugs and anesthetic painkillers are commonly used in drug therapy. However, taking these drugs may also cause gastrointestinal damage, heart and kidney load, and other side effects [10].

At present, there are no nonspecific, safe, and effective drugs and methods in clinical treatment [9], so there is an urgent need to seek a breakthrough from traditional Chinese medicine (TCM). From ancient times to the present, the use of traditional Chinese medicines (TCMs) and all kinds of TCM therapy has played an inestimable role in the treatment of $\mathrm{OA}$ of which experience and therapeutic effects have been tested and refined in Asian countries in the past few thousand years. OA belongs to the category of $\mathrm{Gu} \mathrm{Bi}$ in TCM. In ancient times, medical saints studied OA and recorded its pathogenesis and treatment in many TCM medical works, such as "Inner Classic of the Yellow Emperor" and "Treatise on Febrile and Miscellaneous Diseases." The most important thing is to record the excellent results of TCM in the treatment of OA [11]. The treatment of OA with TCM includes internal and external use of TCMs, acupuncture (ACU), massage manipulation and Tai Chi exercise, and other therapies. Various studies have shown that the use of TCMs has analgesic and anti-inflammatory effects on the treatment of OA. ACU is one of the most common complementary and alternative medical therapies in medicine and is also a popular therapy for relieving pain and treating dysfunction related to musculoskeletal conditions. The therapeutic effect of ACU will remain unchanged with the passage of time [12]. The use of a massage technique can effectively reduce the pain of each joint and achieve the purpose of supplementary treatment. Tai Chi exercise can improve the physical and mental health of patients and effectively relieve psychological depression symptoms caused by OA pain. This article mainly introduces the therapeutic effects of oral administration of TCMs (OATCM), external use of TCMs (EUTCM), and ACU on OA.

Here, we provide a novel and comprehensive meta analysis with detailed information for the efficacy of TCM including TCMs and ACU on patients with OA (Figure 1).

\section{Methods and Program}

As Dr. zou et al., 2019 [13], have done a lot of solid data retrieval work using various databases, their data mining and processing methods are worth learning and using for reference. Therefore, we followed the methods of Dr. Zou et al., 2019.

2.1. Literature Retrieval Strategy. Keywords "Osteoarthritis (OA)" [Title/Abstract] AND "Traditional Chinese medicine (TCM)" OR "Acupuncture (ACU)" [Title/Abstract] AND "Clinical" [Title/Abstract] were used as search items in electronic databases including Pubmed, Wanfang, CNKI, CBM, and VIP. Articles published before August 1, 2019, were checked without language restrictions in order to obtain a comprehensive search. All relevant articles were downloaded into Endnote software (version X9, Thomson Reuters, Inc., New York, NY, United States) for exploring further. Duplicate records were removed. A full-text review was performed while the title/abstract were thought to be thematic. The job mentioned above was executed by three investigators independently. Conflicts were resolved by the consensus and discussion.

2.2. Inclusion and Exclusion Criteria. According to the suggestions of medical experts, we designed the inclusion criteria as follows: (1) Patients in RCTs were diagnosed with OA by diagnostic criteria of knee osteoarthritis (DCKO), guidelines for the diagnosis and treatment of osteoarthritis (GDTO) version 2007, guiding principles for clinical research of new drugs of traditional Chinese medicine (GPCRNDTCM), clinical guideline of new drugs for traditional Chinese medicine (CGNDTCM), diagnosis and treatment scheme of traditional Chinese medicine for 95 diseases in 22 specialties (DTSTCM95D22S), criteria for diagnosis and therapeutic effect of diseases and syndromes of traditional Chinese medicine (CDTEDSTCM), or diagnostic criteria of therapeutic effect of traditional Chinese medicine on knee osteoarthritis (DCTETCMKO). (2) All trials mentioned were described as RCTs. (3) Patients in the most of the experimental groups received TCM therapy, whereas patients in the most of the control groups received Western medicine therapy. (4) Outcome measurements of each study must have included a minimum of one of the following indices: total effective rate (TER), Western Ontario and McMaster University osteoarthritis index (WOMAC), visual analogue scale (VAS), interleukin (IL)-1, IL-6, tumor necrosis factor- $\alpha$ (TNF- $\alpha$ ), syndrome score of traditional Chinese medicine (SSTCM), erythrocyte sedimentation rate (ESR), $C$-reactive protein (CRP), matrix metalloproteinases3 (MMP-3), insulin-like growth factor-1 (IGF-1), bone morphogenetic protein-7 (BMP-7), receptor activator of nuclear factor- $\kappa$ B ligand (RANKL), bone gla protein (BGP), osteoprotegerin (OPG), fibroblast growth factor-2 (FGF-2), angiopoietin I (Ang I), vascular endothelial growth factor (VEGF), recurrence rate (RR), swelling score (SS), superoxide dismutase (SOD), transforming growth factor- $\beta$ (TGF- $\beta$ ), Lysholm, and Lequesne. 


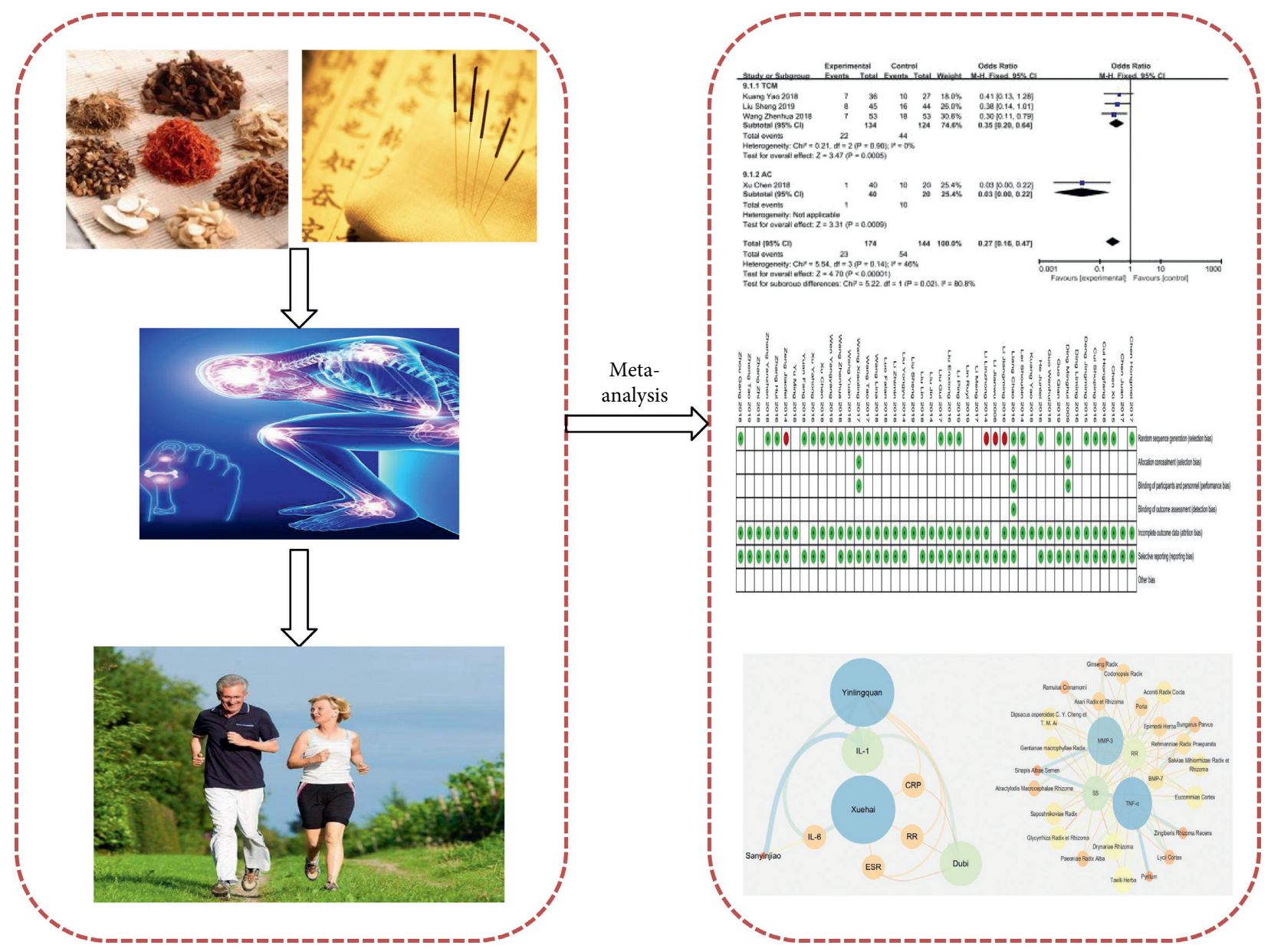

FIGURE 1: Work flow of the present study.

The exclusion criterion was designed as follows: (1) Articles such as reviews, animal trials, case report, and comments were thought to be unrelated to the topic. (2) Trials were not RCTs, or diagnostic criteria in the statement were ambiguous. (3) The intervention of OA patients was not based on TCM treatment.

\subsection{Data Extraction and Quality Assessment. Information on} qualified studies, including authors, methods, sample size, interventions, and result measurements is obtained. The quality of the included study was independently evaluated by three researchers according to the Cochrane Handbook for Systematic Reviews of Interventions, and different opinions were resolved by consensus. Quality assessment includes random sequence generation (selection bias), allocation concealment (selection bias), blindness of participants and personnel (performance bias), blindness of result evaluation (detection bias), incomplete result data (attrition bias), selective reporting (reporting bias), and other deviations. There are three levels of judgment each semester. The "low risk" of the deviation indicates that the description of the method or procedure is appropriate, the "high risk" indicates that the description of the method or procedure is insufficient or incorrect, and the "unclear risk" indicates that the description of the method and/or procedure is insufficient.
2.4. Data Analysis. Data analysis was performed using Review Manager 5.3 (Cochrane Collaboration). Outcome measures such as TER and RR were regarded as dichotomous variables and presented as the odds ratio (OR) with $95 \%$ confidence intervals (95\% CI), contents of inflammatory cytokines (IL-1, IL-6, TNF- $\alpha$, MMP-3, and TGF- $\beta$ ), indexes of scoring (SSTCM, VAS, WOMAC, Lysholm, SS, and Lequesne), and levels of bone metabolism (IGF-1, OPG, BGP, FGF-2, RANKL, and BMP-7), and factors of ESR and CRP were continuous variables that presented as the mean difference (MD) with 95\% CI. $P$ statistics and $I^{2}$ tests were applied to assess heterogeneity among studies. A fixed-effect model was used to analyze data with low heterogeneity $\left(P>0.1\right.$ and $\left.I^{2} \leq 50 \%\right)$, and data with high heterogeneity $\left(P<0.1\right.$ or $\left.I^{2}>50 \%\right)$ were estimated using the random-effects model. Potential publication bias was revealed by funnel plots.

\section{Results}

3.1. Characteristics of the Eligible Studies. A total of 7836 studies were identified through database retrieval, of which 4260 were deleted due to duplication. Of the remaining 3575 studies, 3487 were excluded due to substandard topics. After that, there were still 88 articles to be further examined in full. 
Forty-four studies were excluded from this procedure because of unclear diagnosis in 8 articles, inappropriate interventions in 21 studies, and single-arm design in 15 studies. Forty-four studies [14-57] were included in quantitative synthesis finally (Figure 2).

Meta-analysis was performed on 4014 OA patients (2012 in the experimental group and 2002 in the control group). The age of the patients ranged from 40 to 80 years, and there was no obvious difference in terms of age and sex between the two groups (Table 1). Trials were conducted between 2000 and 2019; all were RCTs with a comparison between TCM therapy only and Western medicine therapy or with a comparison between a combination of TCM therapy and Western medicine therapy. The usual regimens of TCM therapy were OATCM, EUTCM, and the ACU. In the EUTCM, it can be divided into the methods of traditional Chinese medicine iontophoresis (TCMI), hot compress of TCMs, and fumigation of TCMs. The usual regimens of Western medicine therapy were sodium hyaluronate $(\mathrm{SH})$, arthroscopic debridement (AD), and Western medicine capsules. Thirty studies reported that the duration of treatment lasted for 2 to 4 weeks. Three trials reported a follow-up ranging from 3 to 6 months (Table 2). The TCMs and ACU involved in each article were integrated. The prescriptions handed down from ancient times to today, including the OATCM and EUTCM group, were sorted out (Table S1). The acupoint involved in the ACU group articles were also integrated (Table S2). At the same time, we provided the corresponding international code for acupoint (Table S3).

3.2. Quality of Included Trials Assessment. According to the Cochrane risk of deviation estimation, all trials in the literature mentioned the random allocation of participants, and 30 trials $[14,16-18,20,21,23-26,28,29,32-34$, $38-46,51-53,55-57]$ described the conditions for the generation of random allocation sequences. Detailed information on allocation concealment and blinding of participants of majority studies was mentioned in 3 trials $[45,56,57]$. Blinding of outcome assessment of all studies was reported in 1 trial [56]. Forty-two studies [14-36, $38-50,52-57]$ were considered to have a lower risk of wear bias due to the availability of complete data. Thirty-nine trials [14-18, 21-24, 27-29, 31-57] reported detailed indicators, thus indicating a lower risk of reporting bias (Figure 3).

\subsection{Outcome Measures with Subgroup Analysis}

3.3.1. TER of TCM Therapy vs. Western Medicine Therapy. The standard of clinical efficacy reported in the included trials was divided into four grades including clinical recovery, marked effect, effectiveness, and invalidation. TER referred to the percentage of patients who were evaluated to recovery, marked effect, and effectiveness. Thirty-seven studies reported TER among which there were ten trials $[14,16,19,20,26,29,39,47,48,51]$ in the OATCM group which meta-analyzed that OATCM treatment significantly improved TER in the treatment of OA $(\mathrm{OR}=2.61,95 \% \mathrm{CI}$ : $1.76,3.85 ; P<0.00001)$ using a fixed-effect model $(P=0.99$, $I^{2}=0 \%$ for heterogeneity test). Seventeen studies $[15,17,22-25,30,33,34,36,38,40,41,43,46,52,55]$ demonstrated that EUTCM treatment significantly improved TER $(\mathrm{OR}=3.69$, 95\%CI: 2.66, 5.12; $P<0.00001)$ using a fixed-effect model $\left(P=0.99, I^{2}=0 \%\right.$ for heterogeneity test). Ten trials $[21,32,35,37,42,44,50,53,56,57]$ mentioned that ACU treatment improved TER significantly $(\mathrm{OR}=4.19,95 \% \mathrm{CI}: 2.72,6.45 ; P<0.00001)$ with a fixedeffect model ( $P=0.99, I^{2}=0 \%$ for heterogeneity test) of meta-analysis. According to the overall analysis of TER, TCM therapy significantly improved the clinical efficiency compared with the control group (Figure 4).

3.3.2. Indicators of Self-Activity Score of TCM Therapy vs. Western Medicine Therapy. VAS, WOMAC, SSTCM, Lysholm, SS, and Lequesne were the important indices mentioned in included studies reflecting self-activity and therapeutic effects.

Seven trials $[14,16,19,20,26,47,51]$ mentioned the determination of VAS in the OATCM group $(\mathrm{MD}=-1.45,95 \%$ CI: $-2.05,-0.85 ; P<0.00001)$ with heterogeneity $(P<0.00001$, $\left.I^{2}=97 \%\right)$. Eleven trials $[15,17,24,30,31,34,36,38,43,49,55]$ recorded VAS in the EUTCM group $(\mathrm{MD}=-1.12,95 \% \mathrm{CI}$ : $-1.58,-0.67 ; P<0.00001)$ with heterogeneity $(P<0.00001$, $\left.I^{2}=95 \%\right)$, and four trials $[21,37,50,53]$ provided VAS in the ACU group ( $\mathrm{MD}=-1.94,95 \% \mathrm{CI}:-2.66,-1.22 ; P<0.00001)$ with heterogeneity $\left(P<0.00001, I^{2}=94 \%\right)$, so a random-effect model was applied to finish the meta-analysis mentioned above. The result of meta-analysis demonstrated that the TCM therapy significantly decreased the level of VAS $(\mathrm{MD}=-1.39,95 \% \mathrm{CI}$ : $-1.74,-1.05 ; P<0.00001$; Figure 5(a)).

In terms of WOMAC index, it was reported in the OATCM group, EUTCM group, and ACU group. Eight trials $[14,19,20,26,29,47,48,51]$ mentioned the WOMAC in the OATCM group ( $\mathrm{MD}=-9.92,95 \% \mathrm{CI}:-14.33,-5.50$; $P<0.0001)$ with heterogeneity $\left(P<0.00001, I^{2}=96 \%\right)$. Eight trials $[17,18,25,30,34,41,52,55]$ recorded WOMAC in the EUTCM group $(\mathrm{MD}=-5.35, \quad 95 \% \mathrm{CI}:-8.14, \quad-2.56$; $P=0.0002)$ with heterogeneity $\left(P<0.00001, I^{2}=96 \%\right)$, and eight trials $[21,27,28,32,35,45,54,57]$ reported in the ACU group ( $\mathrm{MD}=-16.24,95 \% \mathrm{CI}:-29.82,-2.65 ; P=0.02)$ with heterogeneity $\left(P<0.00001, I^{2}=100 \%\right)$, so a random-effect model was applied to finish the meta-analysis mentioned above which showed that the TCM therapy significantly decreased the level of WOMAC compared with the control group $(\mathrm{MD}=-10.49,95 \% \mathrm{CI}:-14.68,-6.31 ; P<0.00001$; Figure 5(b)).

In terms of the Lysholm score, seven trials $[15,18,30,31,36,38,49]$ recorded Lysholm in the EUTCM group $(\mathrm{MD}=9.42,95 \% \mathrm{CI}: 6.33,12.52 ; P<0.00001)$ with heterogeneity $\left(P<0.00001, I^{2}=83 \%\right)$ and three trials $[21,50,53]$ provided Lysholm in the ACU group $(\mathrm{MD}=23.04,95 \% \mathrm{CI}: 13.59,32.49 ; P<0.00001)$ with heterogeneity $\left(P<0.00001, I^{2}=90 \%\right)$, so a random-effect model was applied to finish the meta-analysis mentioned above which demonstrated that the TCM therapy significantly 


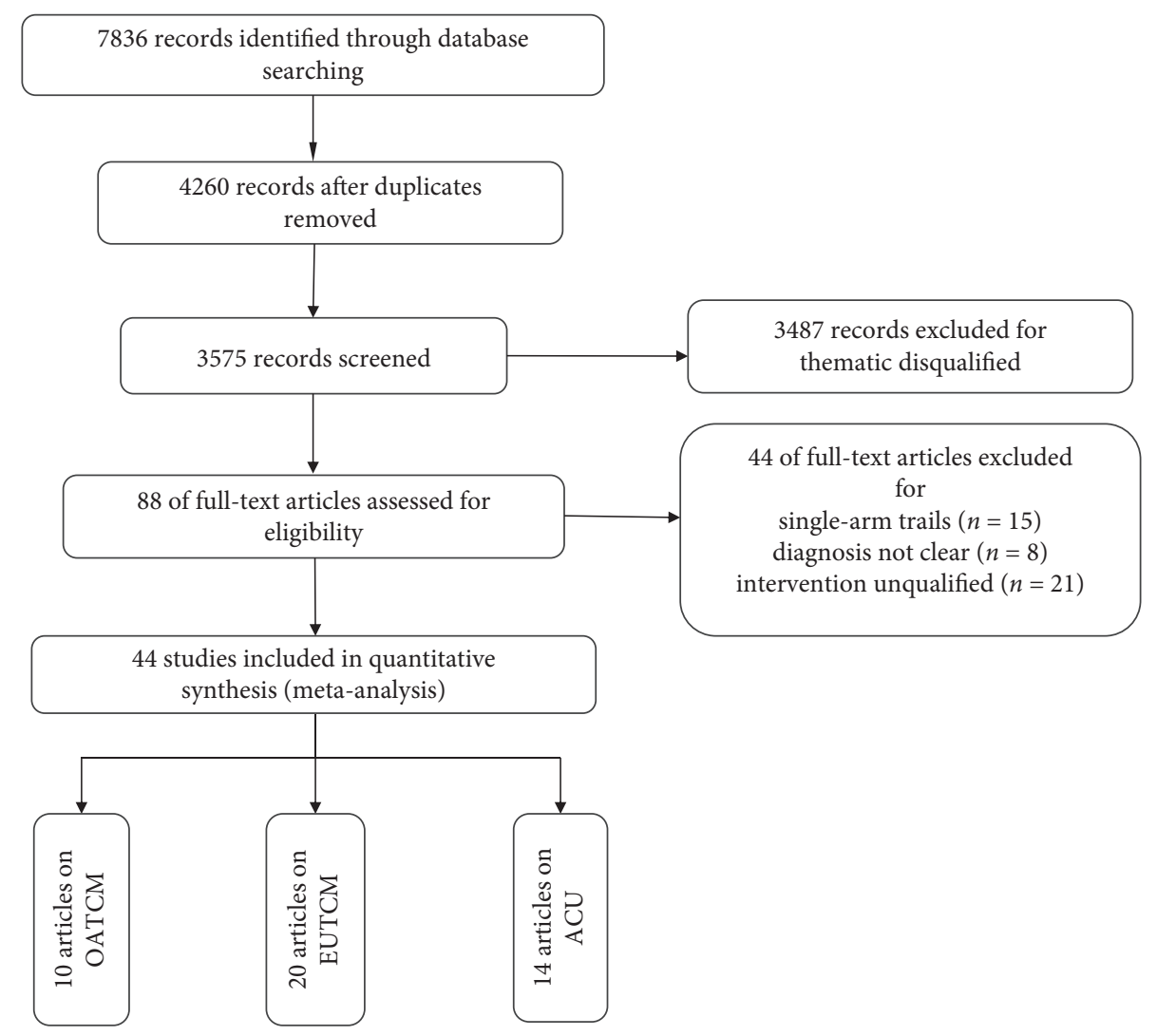

FIgURe 2: Process of the study extracted for meta-analysis.

increased the level of Lysholm $(\mathrm{MD}=13.93,95 \% \mathrm{CI}: 8.71$, 19.15; $P<0.00001$; Figure 5(c)).

In terms of the SSTCM, three trials $[16,39,51]$ recorded SSTCM in the OATCM group $(\mathrm{MD}=-4.01,95 \% \mathrm{CI}:-5.15$, -2.86; $P<0.00001)$ with heterogeneity $\left(P=0.01, I^{2}=76 \%\right)$ and three trials $[33,52,55]$ provided SSTCM in the EUTCM group ( $\mathrm{MD}=-1.95,95 \% \mathrm{CI}:-3.74,-0.17 ; P=0.03)$ with heterogeneity $\left(P=0.005, I^{2}=81 \%\right)$, so a random-effect model was applied to finish the meta-analysis mentioned above which showed that the TCM therapy significantly decreased the level of SSTCM $(\mathrm{MD}=-3.06,95 \% \mathrm{CI}:-4.16$, $-1.95 ; P<0.00001$; Figure 5(d)).

3.3.3. Inflammatory Cytokines of TCM Therapy vs. Western Medicine Therapy. Inflammatory cytokines play an important role in the occurrence and development of OA. Inflammatory indices reported in eligible studies included IL-1, IL-6, TNF- $\alpha$, MMP-3, and TGF- $\beta$. For the IL-1 index, three studies $[14,20,29]$ reported in the OATCM group with meta-analysis that OATCM significantly decreased the level of IL-1 (MD $=-11.54,95 \% \mathrm{CI}$ : $-25.51,2.42)$ with a randomeffect model because of heterogeneity existence $(P<0.00001$, $\left.I^{2}=99 \%\right)$. Ten articles $[18,22,23,25,30,31,36,40,43,46]$ proved that EUTCM therapy significantly decreased the level of IL-1 (MD $=-11.07,95 \% \mathrm{CI}:-14.22,-7.91$; $P<0.00001)$ using a random-effect model because of heterogeneity existence $\left(P<0.00001, I^{2}=99 \%\right)$. Four trials $[21,28,32,35]$ mentioned that ACU therapy can significantly decrease the IL-1 $(\mathrm{MD}=-14.57$, 95\%CI: $-23.29,-5.84 ; P=0.001)$ with a random-effect model $\left(P<0.00001, I^{2}=99 \%\right.$ for the heterogeneity test $)$ of metaanalysis (Figure 6(a)).

Three studies $[14,16,29]$ in the OATCM group, eleven trials $[18,22,23,25,30,31,36,38,40,41,43]$ in the EUTCM group, and five trials $[21,28,32,35,50]$ in the ACU group reported that TCM therapy reduced the level of TNF- $\alpha$ significantly of which $\mathrm{MD}$ with $95 \% \mathrm{CI}$ was $((\mathrm{MD}=-1.29$, 95\%CI: $-2.44, \quad-0.13 ; \quad P=0.03), \quad(\mathrm{MD}=-15.73$, 95\%CI: $-22.17,-9.29 ; P<0.00001)$, and $(\mathrm{MD}=-1.10,95 \% \mathrm{CI}:-1.51$, $-0.68 ; P<0.00001))$ meta-analyzed by the random-effect model $\left(P<0.00001, I^{2}=99 \%\right.$ for the heterogeneity test, respectively) (Figure 6(b)).

Five studies $[14,16,20,26,39]$ of OATCM, four trials $[15,40,46,49]$ of EUTCM, and one article [27] of the ACU group mentioned the MMP-3. The MD with 95\% CI for MMP-3 was $((\mathrm{MD}=-19.33,95 \% \mathrm{CI}:-27.95,-10.70)$, $(\mathrm{MD}=-2.58,95 \% \mathrm{CI}:-4.18,-0.98)$, and $(\mathrm{MD}=-25.44,95 \%$ CI: $-30.28,-20.60)$ ) certified a significant decrease in the experimental group compared with control group $(P<0.0001$; Figure 6(c)).

3.3.4. Influence on Other Indices of TCM Therapy vs. Western Medicine Therapy. In this meta-analysis, we summarized 5 and less articles related to the same index and summarized the results in the supplemental files. OATCM therapy can significantly reduce SS $(P<0.0001)$ and Lequesne 
TABLE 1: Characteristics of eligible studies.

\begin{tabular}{|c|c|c|c|c|c|c|c|}
\hline & Author, year & Cases $T / C$ & Diagnostic standard & Age (years) & range, mean & Sex ma & e/female \\
\hline & Wang Zhenhua, 2018 & $53 / 53$ & GDTO and GPCRNDTCM & T: $41-72,50.17$ & C: $43-74,51.49$ & $\mathrm{~T}: 29 / 24$ & C: $31 / 22$ \\
\hline & Kuang Yao, 2018 & $49 / 42$ & GDTO and DTSTCM95D22S & T: 56.13 & C: 55.65 & $\mathrm{~T}: 25 / 24$ & C: $20 / 22$ \\
\hline & Wen Yangyang, 2019 & $63 / 63$ & GDTO and GPCRNDTCM & $\mathrm{T}: 61.98$ & C: 62.61 & $\mathrm{~T}: 32 / 31$ & C: $36 / 27$ \\
\hline & Cui Hongfang, 2018 & $61 / 61$ & GDTO and GPCRNDTCM & $\mathrm{T}: 45-76,64.7$ & C: $46-75,62.5$ & $\mathrm{~T}: 31 / 31$ & C: $32 / 29$ \\
\hline & Zheng Tao, 2019 & $50 / 50$ & GDTO and CDTEDSTCM & T: $52-67,63.26$ & C: $51-67$ & $\mathrm{~T}: 27 / 23$ & C: $24 / 26$ \\
\hline ОАТСM & Yuan Fang, 2018 & $30 / 30$ & GDTO (2007) and CDTEDSTCM & $\mathrm{T}: 42-72,54.47$ & C: $41-72,60.63$ & $\mathrm{~T}: 4 / 26$ & C: $6 / 24$ \\
\hline & Li Zhimin, 2018 & $48 / 48$ & DCKO and CDTEDSTCM & $\mathrm{T}: 47-78,57.42$ & C: $45-76,58.24$ & T: $29 / 19$ & C: $31 / 17$ \\
\hline & Liu Sheng, 2019 & $50 / 50$ & GDTO (2010) and TCMDTS95D22S & T: $54-70,58.34$ & C:53-71, 57.43 & $\mathrm{~T}: 25 / 25$ & C: $26 / 24$ \\
\hline & Li Ping, 2019 & $74 / 74$ & CETCMDTKO & $\mathrm{T}: 47-73,60.69$ & C: $45-75,59.48$ & $\mathrm{~T}: 24 / 50$ & C: $25 / 49$ \\
\hline & Li Linzhong, 2014 & $40 / 40$ & NR & $\mathrm{T}: 42-75,57.43$ & $\mathrm{C}: 41-74,56.31$ & $32 / 48$ & - \\
\hline & Yu Ming, 2018 & $73 / 73$ & DCKO (2007) & $43-80,54.86$ & - & T: $40 / 33$ & C: $46 / 27$ \\
\hline & Li Meng, 2017 & $30 / 30$ & DCKO & $\mathrm{T}: 41-61,52.41$ & C: $42-60,51.27$ & $\mathrm{~T}: 13 / 17$ & C: $12 / 18$ \\
\hline & Lei Shoubin, 2014 & $49 / 49$ & GDTO & $\mathrm{T}: 43-70,57.5$ & C: $44-71,58.7$ & $\mathrm{~T}: 17 / 32$ & C: $18 / 31$ \\
\hline & Zeng Jiaofei, 2014 & $50 / 50$ & DCKO and $\mathrm{K}-\mathrm{L}$ & $\mathrm{T}: 45-63,56.1$ & C: $45-65,55.6$ & $\mathrm{~T}: 21 / 29$ & C: $23 / 27$ \\
\hline & He Junlei, 2016 & $57 / 55$ & DCKO & $\mathrm{T}: 45-$ & C: $43-75,59.06$ & $\mathrm{~T}: 16 / 41$ & C:19/36 \\
\hline & Chen Xi, 2015 & $40 / 40$ & DCKO and GDTO & $40-7$ & - & $35 / 45$ & - \\
\hline & Liu Enxiong, 2016 & $68 / 68$ & GDTO and GPCRNDTCM & $\mathrm{T}: 53-79,61.29$ & C: $51-78,61.01$ & $\mathrm{~T}: 45 / 23$ & C: $48 / 20$ \\
\hline & Zhou Gang, 2018 & $38 / 38$ & GDTO (2007) and CDTEDSTCM & $\mathrm{T}: 46-70,55.62$ & C: $45-68,54.33$ & $\mathrm{~T}: 16 / 22$ & C: $20 / 18$ \\
\hline & Lui Gui, 2017 & $60 / 60$ & GDTO and GPCRNDTCM & T: $59-72,64.14$ & C: $58-74,64.29$ & T: $19 / 41$ & C: $21 / 39$ \\
\hline & Ding Liming, 2016 & $51 / 47$ & $\mathrm{NR}$ & $\mathrm{T}: 63.00$ & C: 67.08 & $\mathrm{~T}: 16 / 35$ & C: $14 / 33$ \\
\hline EUTCM & Zhang Yanzhen, 2018 & $34 / 34$ & CDTEDSTCM and DCKO (1995) & $\mathrm{T}: 42-73,52.34$ & C: $42-73,54.24$ & $\mathrm{~T}: 12 / 22$ & C: $10 / 24$ \\
\hline & Liu Yongyu, 2014 & & & $\mathrm{~T}: 51-69$ & C: $51-69$ & NR & NR \\
\hline & Wang Yua & & DCKO (1995) & $\mathrm{T}: 40-$ & C:44-73, & T: $8 / 22$ & C: $2 / 28$ \\
\hline & Zhang Zhi, 2018 & $52 / 51$ & GDTO (2007) and K-L & T: $55-68,63.9$ & C: $53-70,64.8$ & T: $24 / 28$ & C: $24 / 27$ \\
\hline & Chen Hongmei, 2017 & $31 / 30$ & DCKO & T: 58.18 & C:55.90 & $\mathrm{T}: 13 / 18$ & C: $11 / 19$ \\
\hline & Zhang Hui, 2016 & $51 / 51$ & PO and TCMDTS95D22S & $\mathrm{T}: 41-79,57.2$ & C: $40-78,56.1$ & $\mathrm{~T}: 17 / 34$ & C:19/32 \\
\hline & Liu Lin, 2018 & $40 / 40$ & DCCASTCM & $\mathrm{T}: 40-70$ & C: $40-70$ & NR & NR \\
\hline & Cui Shuping, 2016 & $35 / 35$ & GDTO and GPCRNDTCM & $\mathrm{T}: 42-78$ & C: $46-80$ & $\mathrm{~T}: 14 / 21$ & C: $12 / 20$ \\
\hline & Wang Tao, 2017 & $30 / 30$ & $\begin{array}{c}\text { GPCRNDTCM and GDTO } \\
\text { \&TCMDTS95D22S }\end{array}$ & $\mathrm{T}: 42-57,51.1$ & C: $43-56,50.2$ & T: $8 / 22$ & C: $9 / 21$ \\
\hline & Guo Wenhui, 2018 & $45 / 45$ & DCKA $(2007)$ & T: 73.4 & C: 72.7 & T: $25 / 20$ & C: $21 / 24$ \\
\hline & Chen Juan, 2017 & & GDTO (2007) and GPCRNDTCM & T: $45-76,58.51$ & C: $47-80,59.23$ & $\mathrm{~T}: 25 / 21$ & C: $27 / 19$ \\
\hline & Deng Jingming, 2015 & $40 / 40$ & GDTO (2007) & $\mathrm{T}: 60.0$ & C: 62.0 & $\mathrm{~T}: 17 / 23$ & C: $15 / 25$ \\
\hline & Ding Minghui, 2009 & $30 / 30$ & DCKO (1986) & T: 59.37 & C: 58.93 & $\mathrm{~T}: 8 / 22$ & $\mathrm{C}: 8 / 22$ \\
\hline & Guo Qian, 2019 & $79 / 79$ & GDTO and DCTETCMKO & T: $45-78,56.06$ & C:45-78, 56.06 & $\mathrm{~T}: 38 / 41$ & C: $38 / 41$ \\
\hline & Li Jianwu, 2008 & $30 / 30$ & DCKO (1995) & T: 53.0 & C: 55.6 & $\mathrm{~T}: 11 / 19$ & C:9/21 \\
\hline & Li Jiangming, 2016 & $47 / 60$ & NR & $\mathrm{T}: 65.12$ & C: 64.26 & $\mathrm{~T}: 5 / 42$ & C: $7 / 53$ \\
\hline & Liang Chao, 2016 & $30 / 30$ & GDTO (2007) & $\mathrm{T}: 61.4$ & C: 60.3 & $\mathrm{~T}: 22 / 8$ & C: $21 / 9$ \\
\hline $\mathrm{ACO}$ & Lin Ruyi, 2019 & $43 / 43$ & GDTO (2007) & T: $40-69,60.05$ & C: $40-69,59.52$ & $\mathrm{~T}: 22 / 21$ & C: $24 / 19$ \\
\hline & Liu Jin, 2014 & $30 / 30$ & GDTO $(2007)$ & T: 60.8 & C: 62.5 & $\mathrm{~T}: 12 / 18$ & C: $13 / 17$ \\
\hline & Luo Falan, 2018 & $42 / 42$ & GDTO (2007) and GPCRNDTCM & T: $49-67,59.27$ & C: $51-68,58.45$ & $\mathrm{~T}: 18 / 24$ & C: $19 / 23$ \\
\hline & Wang Lina, 2018 & $59 / 59$ & $\mathrm{NR}$ & $\mathrm{T}: 40-75,52.13$ & C: $41-77,53.47$ & $\mathrm{~T}: 24 / 35$ & C: $25 / 34$ \\
\hline & Wang Xiaoling, 2017 & $25 / 21$ & DCKO (1995) & T: $50-74,61$ & C: $44-75,58$ & T: $8 / 17$ & C:2/19 \\
\hline & Xu Chen, 2018 & $45 / 41$ & DCKO (1995) & $\mathrm{T}: 61.0$ & C: 61.0 & $\mathrm{~T}: 24 / 21$ & C: $25 / 16$ \\
\hline & Xu Yahong, 2016 & $54 / 54$ & GDTO and GPCRNDTCM & $\mathrm{T}: 45-73,54.3$ & C: $45-75,55.2$ & $\mathrm{~T}: 25 / 29$ & C: $24 / 30$ \\
\hline
\end{tabular}

$C$, control group; CDTEDSTCM, criteria for diagnosis and therapeutic effect of diseases and syndromes of traditional Chinese medicine; DCKO, diagnostic criteria of knee osteoarthritis; DTSTCM95D22S, diagnosis and treatment scheme of traditional Chinese medicine for 95 diseases in 22 specialties; DCTETCMKO, diagnostic criteria of therapeutic effect of traditional Chinese medicine on knee osteoarthritis; GDTO, guidelines for the diagnosis and treatment of osteoarthritis; GPCRNDTCM, guiding principles for clinical research of new drugs of traditional Chinese medicine; K-L, Kellgren-Lawwrence grading standard; NR, no report; PO, practical orthopaedics; $T$, trial group.

$(P<0.00001)$ levels in self-activity indicators of patients with OA. Also, EUTCM therapy can also reduce Lequesne $(P<0.0001)$, but the therapeutic effect of SS $(P=0.27)$ has no statistical significance (Table S4). Associated inflammatory factors include TGF- $\beta$ and IL-6. OATCM treatment could significantly increase the level of TGF- $\beta(P<0.00001)$, but had no statistical significance in reducing IL-6 $(P=0.12)$. Both EUTCM therapy and ACU therapy can significantly increase TGF- $\beta$ and decrease IL-6 levels $(P<0.001)$ (Table S5). Among the indicators related to bone balance, ACU therapy can significantly increase the level of BGP and FGF-2 and reduce RANKL $(P<0.001)$, but there is no statistical significance for the improvement of IGF-1 $(P=0.24)$ and OPG $(P=0.62)$. OATCM therapy can significantly reduce BMP-7 $(P<0.00001)$ (Table S6). ACU therapy can significantly reduce the levels of Ang I and VEGF $(P<0.00001)$ in the collected studies (Table S7). OATCM and EUTCM therapy can effectively reduce the RR and increase SOD $(P<0.00001)$ (Table S8). A forest plot illustrating the results of the analysis of ESR and CRP 
TABLE 2: Intervention characteristics of included studies.

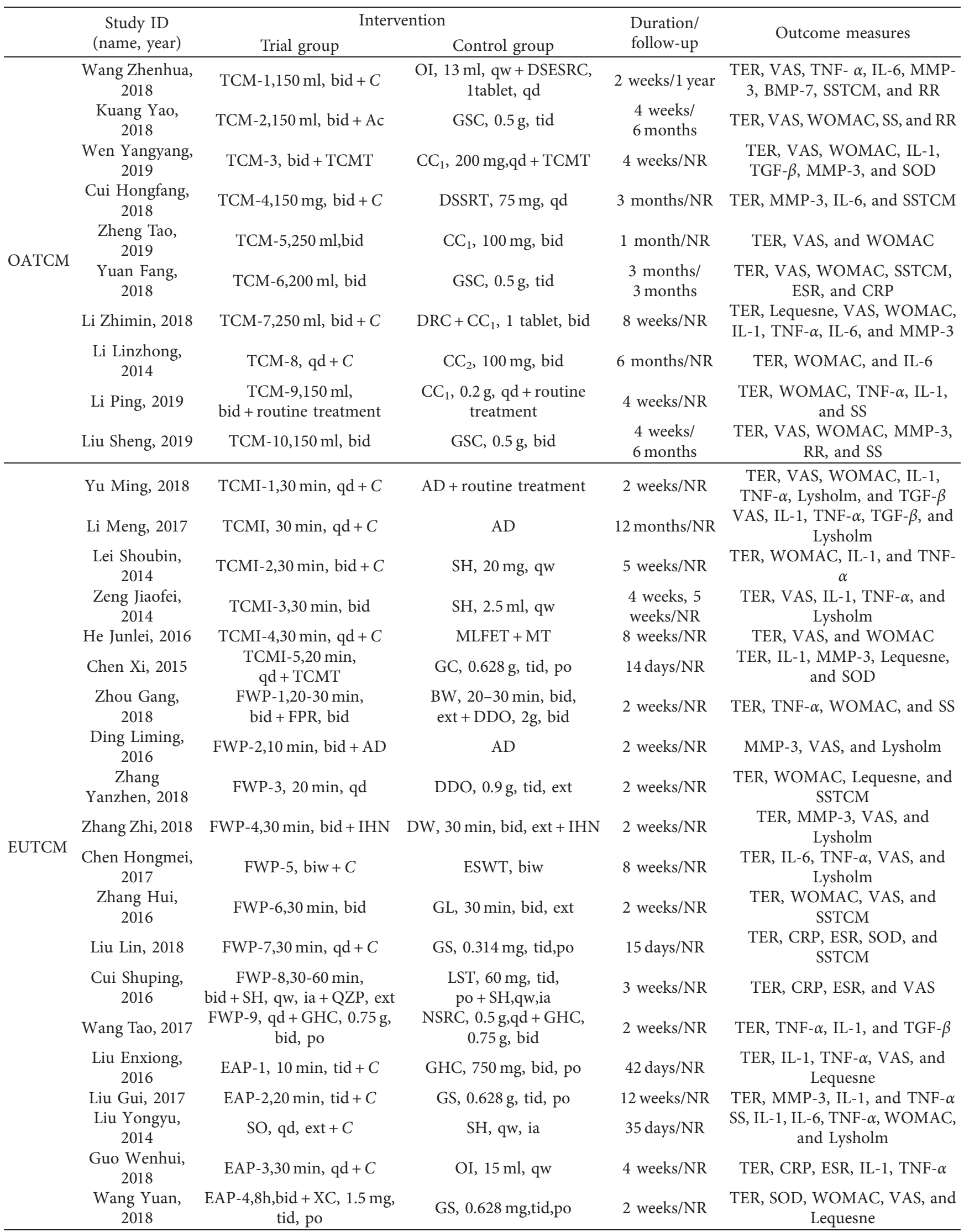


TABLE 2: Continued.

\begin{tabular}{|c|c|c|c|c|c|}
\hline & \multirow{2}{*}{$\begin{array}{c}\text { Study ID } \\
\text { (name, year) }\end{array}$} & \multicolumn{2}{|c|}{ Intervention } & \multirow{2}{*}{$\begin{array}{l}\text { Duration/ } \\
\text { follow-up }\end{array}$} & \multirow{2}{*}{ Outcome measures } \\
\hline & & Trial group & Control group & & \\
\hline \multirow{14}{*}{$\mathrm{ACU}$} & Chen Juan, 2017 & WAC, $\mathrm{qd}+C$ & $\mathrm{SH}$, injection, qw & 4 weeks/NR & TER, VAS, Lysholm, and TNF- $\alpha$ \\
\hline & $\begin{array}{l}\text { Deng Jingming, } \\
2015\end{array}$ & WAC, tiw & ISRC, po, bid & 4 weeks/NR & TER, VAS, and Lysholm \\
\hline & $\begin{array}{l}\text { Ding Minghui, } \\
2009\end{array}$ & WAC, qd & $\mathrm{B}$ & 2 weeks/NR & TER and WOMAC \\
\hline & Guo Qian, 2019 & WAC, qd & B & 3 weeks/NR & $\begin{array}{l}\text { WOMAC, IL- } 1 \text {, TNF- } \alpha \text {, IGF- } 1 \text {, } \\
\text { FGF- } 2 \text {, and TGF- } \beta\end{array}$ \\
\hline & Li Jianwu, 2008 & WAC, qd & XGC, po, bid & 4 weeks/NR & TER and VAS \\
\hline & $\begin{array}{l}\text { Li Jiangming, } \\
2016\end{array}$ & $\mathrm{WAC}+\mathrm{PCB}$ & Routine Treatment & 3 months/NR & WOMAC \\
\hline & $\begin{array}{l}\text { Liang Chao, } \\
2016\end{array}$ & WAC, qd & GHT, po, tid & 4 weeks/NR & TER \\
\hline & Lin Ruyi, 2019 & WAC, qd & DSC, po, bid & 4 weeks/NR & $\begin{array}{c}\text { WOMAC, OPG, MMP-3, FGF- } \\
\text { 2, VEGF, and Ang I }\end{array}$ \\
\hline & Liu Jin, 2014 & ACU + DSTC, tid, po & FSRT, po, qd & 2 weeks/NR & $\begin{array}{c}\text { TER, WOMAC, IL-1, and TNF- } \\
\alpha\end{array}$ \\
\hline & Luo Falan, 2018 & $\mathrm{ACU}, \mathrm{qd}+\mathrm{C}$ & $\mathrm{CC}_{1}, \mathrm{po}, \mathrm{qd}$ & 8 weeks/NR & $\begin{array}{c}\text { TER, IGF-1, RANKL, BGP, and } \\
\text { OPG }\end{array}$ \\
\hline & $\begin{array}{l}\text { Wang Lina, } \\
2018\end{array}$ & WAC, $\mathrm{qd}+C$ & $\mathrm{CC}_{1}$, po, qd & 4 weeks/NR & $\begin{array}{l}\text { TER, WOMAC, IL-1, TNF- } \alpha \text {, } \\
\text { and IL-6 }\end{array}$ \\
\hline & $\begin{array}{l}\text { Wang Xiaoling, } \\
2017\end{array}$ & WAC, qd & $\mathrm{CC}_{1}, 200 \mathrm{mg}$,po & 3 weeks/NR & WOMAC \\
\hline & Xu Chen, 2018 & $\mathrm{WAC}+\mathrm{APP}, \mathrm{qd}$ & SH, qw or GHT, po, bid & $\begin{array}{l}4 \text { weeks/ } \\
3 \text { months }\end{array}$ & TER, CRP, ESR, and RR \\
\hline & $\begin{array}{l}\text { Xu Yahong, } \\
2016\end{array}$ & $\mathrm{WAC}+C, \mathrm{qd}$ & USW, qd & 1 month/NR & $\begin{array}{l}\text { TER, VAS, WOMAC, Lysholm, } \\
\text { IL- } 1 \text {, TNF- } \alpha \text {, BGP, and OPG }\end{array}$ \\
\hline
\end{tabular}

$\mathrm{AD}$, arthroscopic debridement; ACU, acupuncture; APP, auricular point pressing; Ang I, angiopoietin I; BW, boiled water; B, blank; bid, twice a day; BGP, Bone gla protein; BMP-7, bone morphogenetic protein-7; $\mathrm{C}$, treatment of the control group; $\mathrm{CC}_{1}$, velecoxib capsule; $\mathrm{CC}_{2}$, Celebrex capsule; $\mathrm{CRP}$, C-reactive protein; DW, distilled water; DDO, diclofenac diethylamine ointment; DRC, Divinegar Ruiyin capsule; DSESRC, diclofenac sodium enteric sustained release capsule; DSC, diclofenac sodium capsule; DSSRT, diclofenac sodium sustained release tablets; DSTC, dragon and soft-shelled turtle capsule; ext, external use; EUTCM, external use of traditional Chinese medicines; ESWT, extracorporeal shock wave therapy; EAP, external application prescription; ESR, erythrocyte sedimentation rate; FSRT, futalin sustained release tablets; FWP, fumigation and washing prescription; FGF-2, fibroblast growth factor-2; GC, glucosamine capsule; GL, Guyouling liniment; GS, glucosamine sulfate; GSC, glucosamine sulfate capsule; GHC, glucosamine hydrochloride capsule; GHT, glucosamine hydrochloride tablets; IL, interleukin; IGF-1, insulin-like growth factor 1; IHN, internal heat needle; ISRC, ibuprofen sustained release capsule; ia, intraarticular injection; LST, Loxoprofen sodium tablets; MLFET, middle- and low-frequency electric therapy apparatus; MT, mobilization technique; MMP, matrix metalloproteinase; NSRC, naproxen sustained release capsule; NR, no report.; OATCM, oral administration of traditional Chinese medicines; OI, ozone injection; OPG, osteoprotegerin; po, oral administration; QZP, Qizhu Zhanjin Powders; qd, once a day; qw, once a week; RANKL, receptor activator of nuclear factor- $\kappa$ B ligand; RR: recurrence rate; SH, sodium hyaluronate; SO, Shangbai ointment; SSTCM, syndrome score of traditional Chinese medicine; SOD, superoxide dismutase; SH, sodium hyaluronate; SS, swelling score; tid, three times a day; TCM, traditional Chinese medicine; TER, total effective rate; TNF- $\alpha$, tumor necrosis factor- $\alpha$; TGF- $\beta$, transforming growth factor- $\beta$; TCMI, traditional Chinese medicine iontophoresis; traditional Chinese medicine treatment; USW, ultrashort wave; VAS, visual analogue scale; VEGF, vascular endothelial growth factor; WAC, warm acupuncture; WOMAC, Western Ontario and McMaster University osteoarthritis index; XC, Xinfeng capsule; XGC, Xianling Gubao capsule.

indicators is shown in Figure S1. Both OATCM and ACU therapy can effectively reduce ESR and CRP $(P<0.00001)$. In addition, EUTCM therapy can significantly reduce ESR $(P=0.09)$, but there is no statistical significance for CRP $(P=0.15)$.

3.4. Network Relationship Diagram between Acupoints and Corresponding Indexes. The 21 acupoints and 19 related indexes were imported into Cytoscape 3.7.1 software, and the network analysis of the interaction between acupoints and corresponding indexes in ACU treatment of OA was drawn, Figure $7(\mathrm{a})$, through the module analysis of the main graph using the eagle algorithm through ClusterViz plug-in Cytoscape, and three main relational subgraphs were obtained. Figure 7 (a) shows the overall relationship between acupoints and indicators. Figure 7(b) shows the interaction between Yinlingquan (ST9) and Xuehai (SP10) acupoints and inflammatory factors, which have major therapeutic effects. Figure 7(c) mainly shows the effects of acupoint Yanglingquan (GB34), Liangqiu (ST34), Xiyan (EXLE4), Zusanli (ST36), and Heding (EX-LE2) on FGF-2, OPG, and BGP of the bone metabolism indexes. Figure $7(\mathrm{~d})$ shows the effect of acupoints such as Xiyangguan (GB33), Weizhong (BL40), and Ashi on the level of TER, WOMAC, and VAS. After ACU treatment, the indexes have been well improved, and the symptoms of OA have been significantly improved (Figure 7).

3.5. Network Relationship Diagram between TCMs and Corresponding Indexes. The 113 TCMs and 17 related indexes were imported into Cytoscape 3.7.1 software, and the network analysis diagram of the interaction between TCMs 


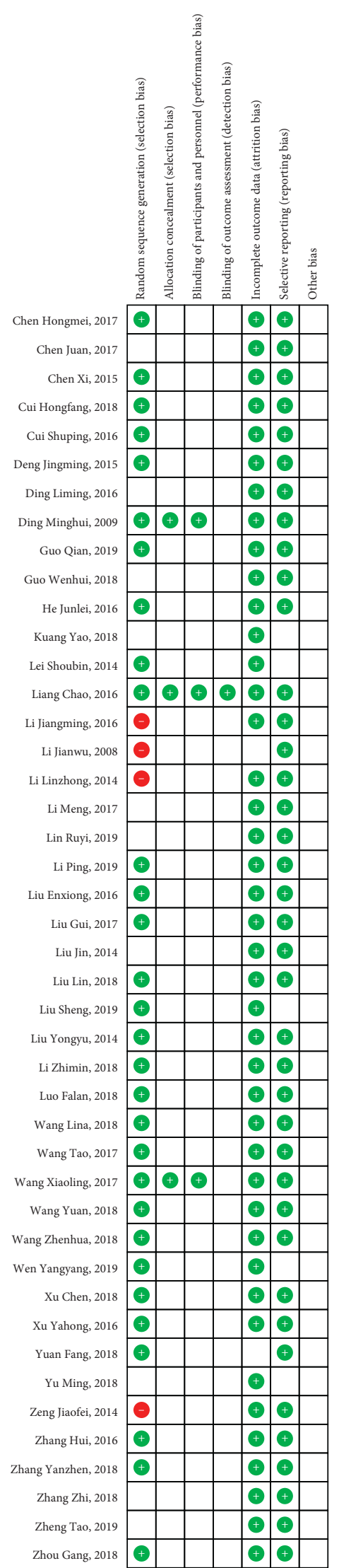

Figure 3: Risk of bias assessment in eligible studies. The quality assessment was conducted by Review Manager 5.3 according to Cochrane Handbook for Systematic Reviews of Interventions Version 5.1.0. Red circle, high risk of bias; green circle, low risk of bias; blank, unclear risk of bias. 


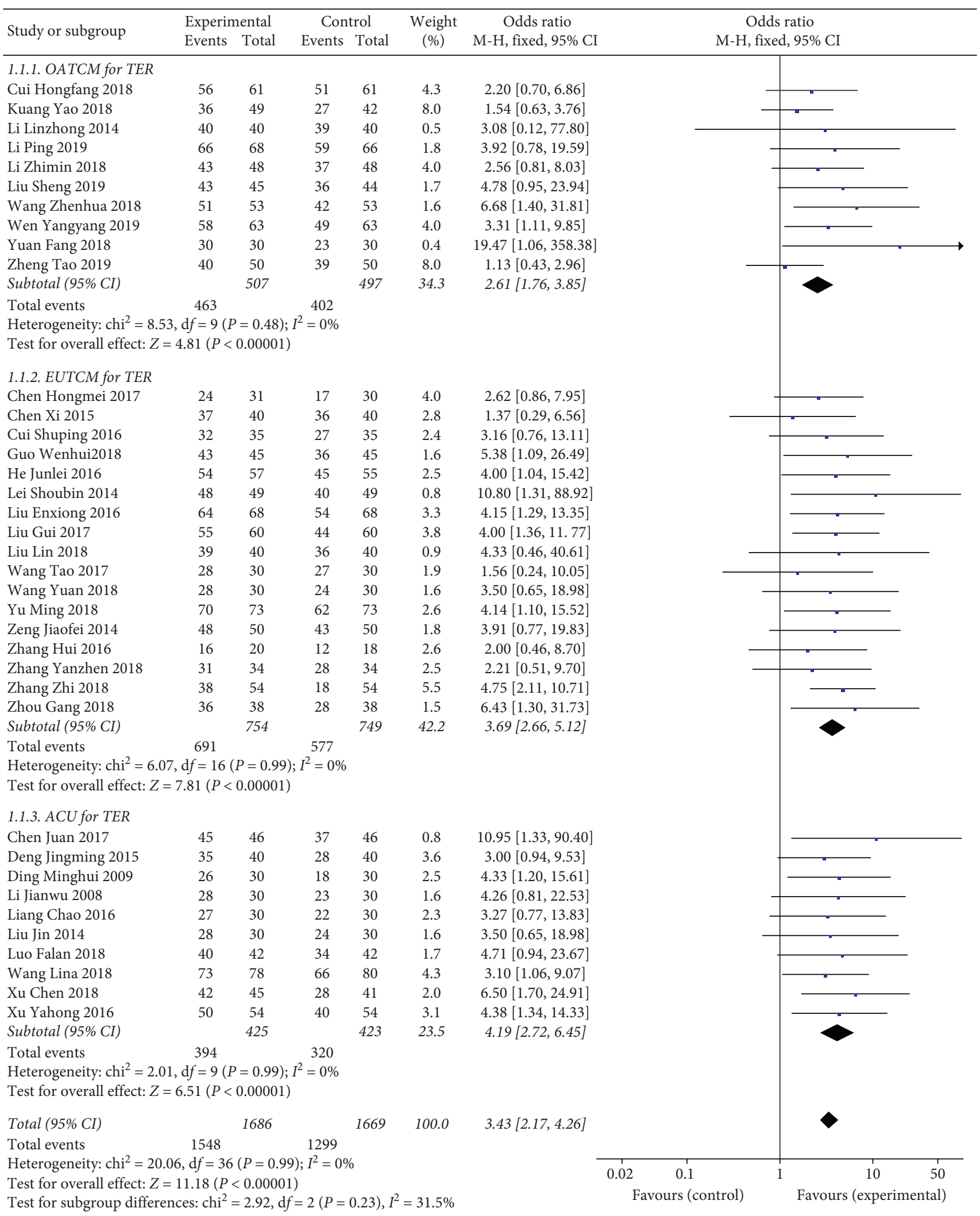

FIGURE 4: Forest plot of TER in patients treated with TCM therapy and Western medicine therapy. I2 and P are the criteria for the heterogeneity test. $\bullet$ : pooled odds ratio, —-—: odds ratio, and $95 \% \mathrm{CI}$. 


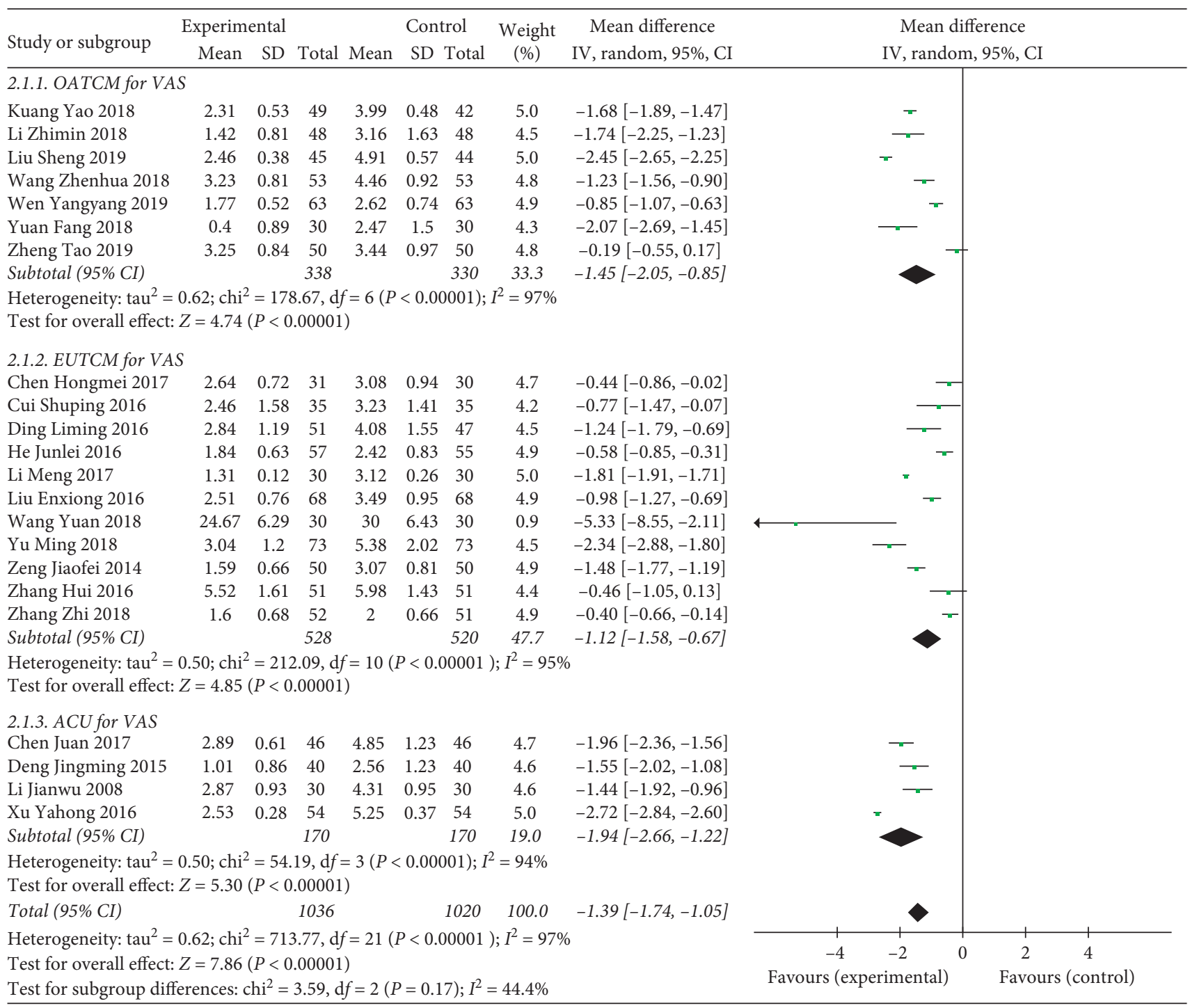

(a)

Figure 5: Continued. 


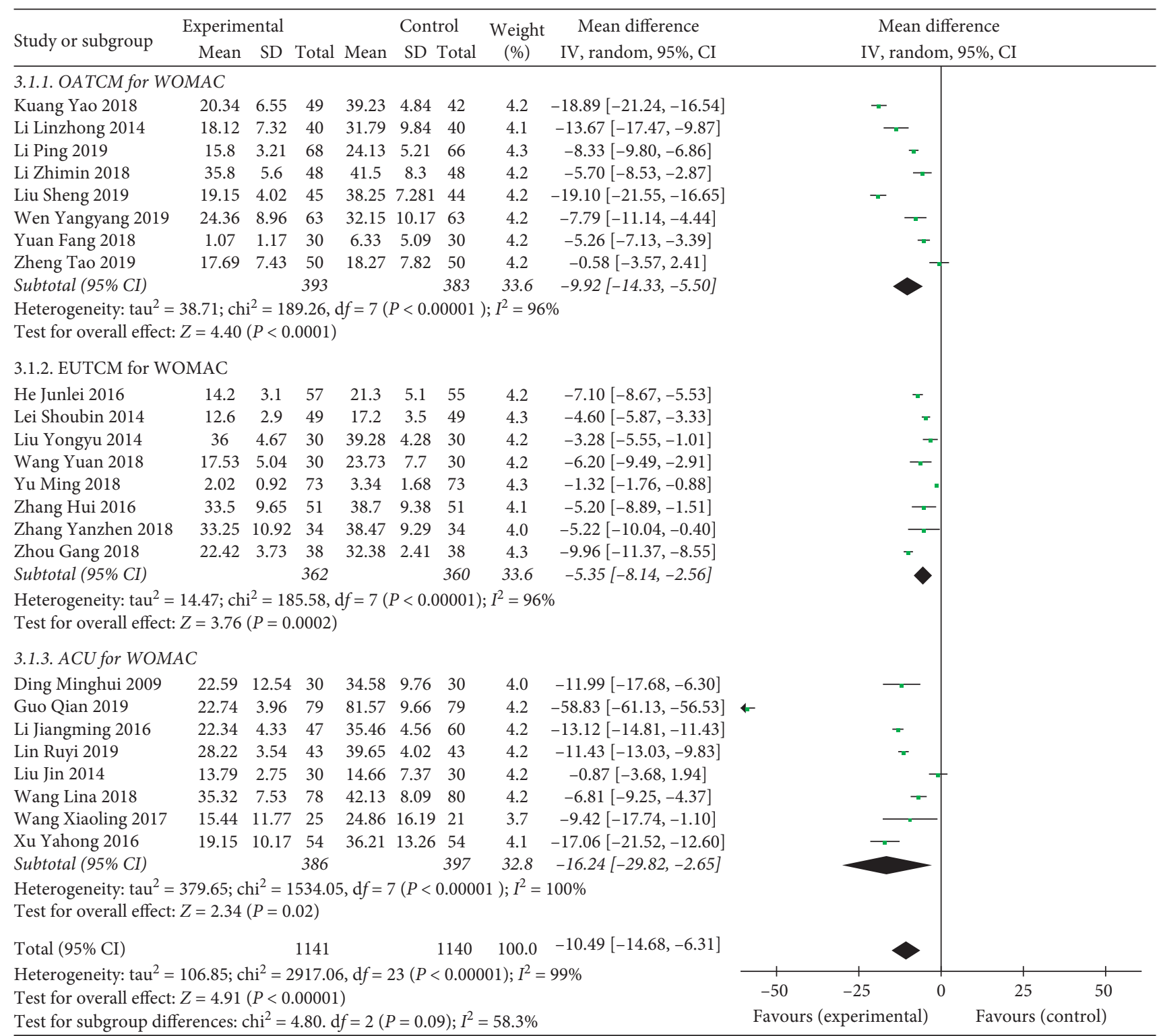

(b)

FIgUre 5: Continued. 


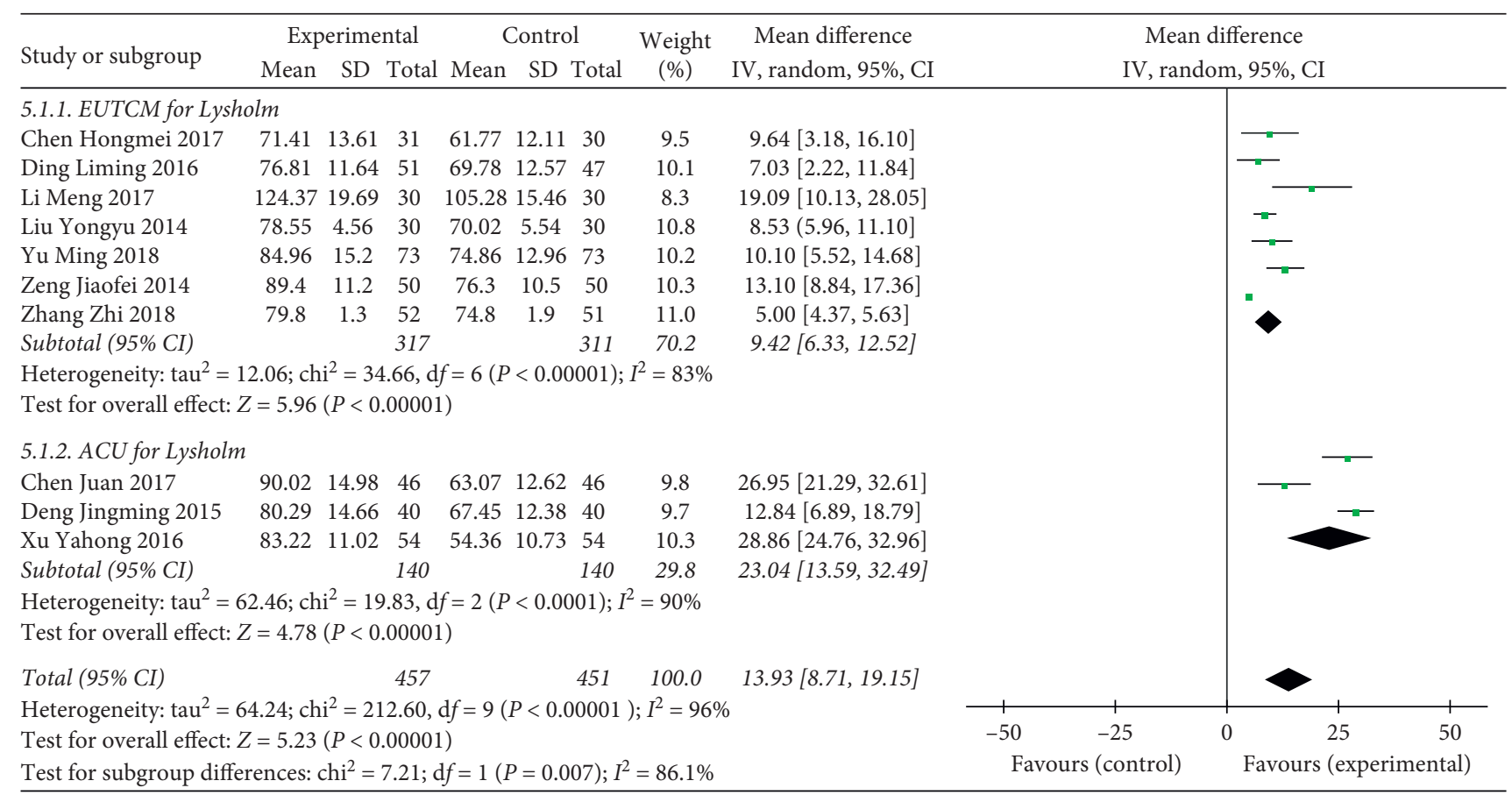

(c)

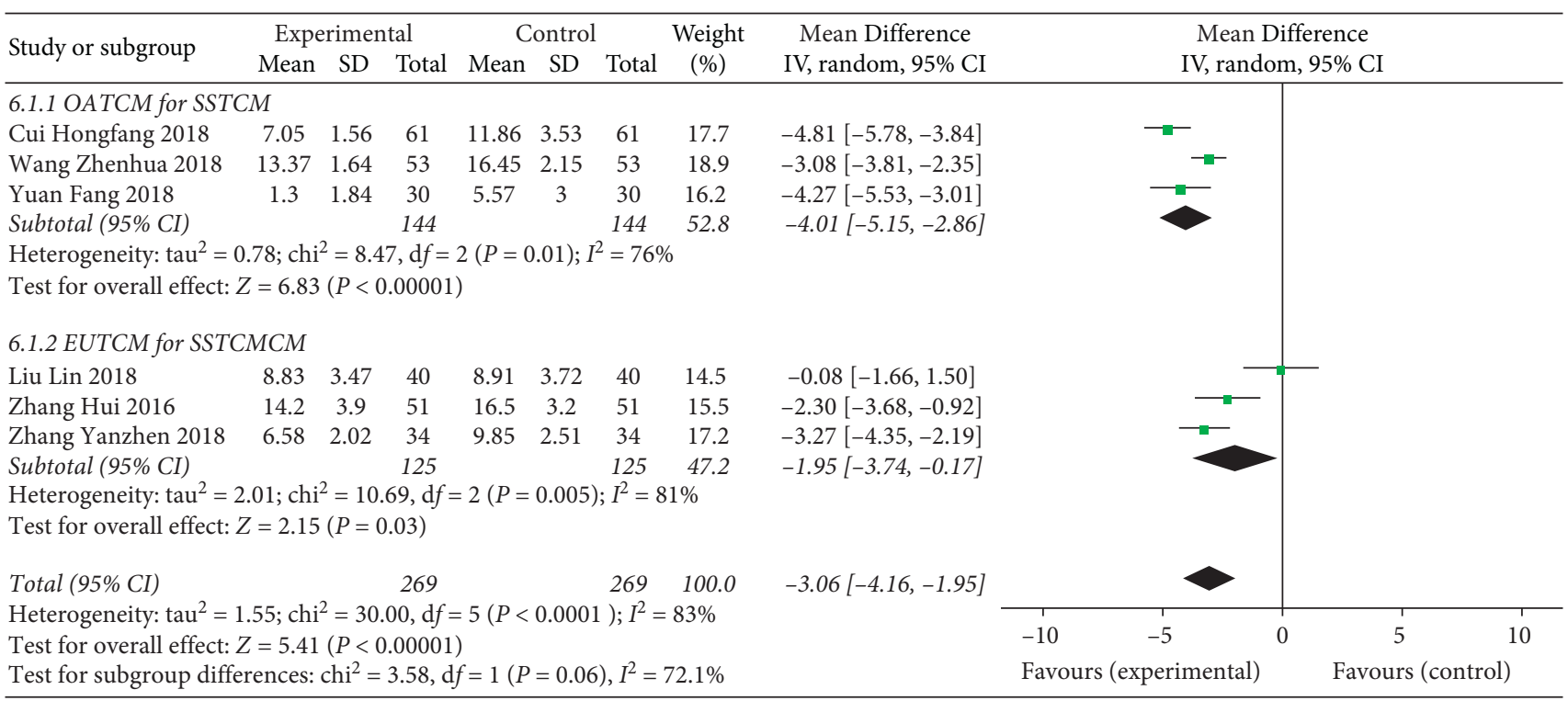

(d)

FIGURE 5: Forest plot of self-activity score in patients treated with TCM therapy and Western medicine therapy. (a) The plot of VAS, (b) the plot of WOMAC, (c) the plot of Lysholm, and (d) the plot of SSTCM. (I) ${ }^{2}$ and (P) are the criteria for the heterogeneity test, $\bullet$ : pooled mean difference, - - - : mean difference, and 95\%CI.

and indicators interaction in the treatment of $\mathrm{OA}$ was drawn, as shown in Figure 8(a). Then, through ClusterViz plug-in Cytoscape, the module analysis of the main graph is carried out by using EAGLE algorithm to get five main relational subgraphs. Figure 8(a) shows the relationship between TCMs and indicators. Figure $8(\mathrm{~b})$ shows that TCMs such as Dipsaci Radix, Aconiti Radix, Typhonii Rhizoma, and Siphonostegiae Herba can ameliorate the score of the pain scale and improve the clinical efficacy.
Figure $8(\mathrm{c})$ shows that Achyranthis Bidentatae Radix, Chuanxiong Rhizoma, Carthami Flos, Angeticae Sinensis Radix, and other drugs can effectively reduce ESR and CRP inflammation indicators ESR and CRP, reduce pain, and improve joint function. Figure 8(d) shows that TCMs such as Drynariae Rhizoma, and Taxilli Herba have a significant relationship with inflammation, reducing SS by reducing TNF- $\alpha$ and MMP-3. Figure 8(e) shows that Rhei Radix et Rhizoma, Lycopi Herba, and Persicae Semen can 


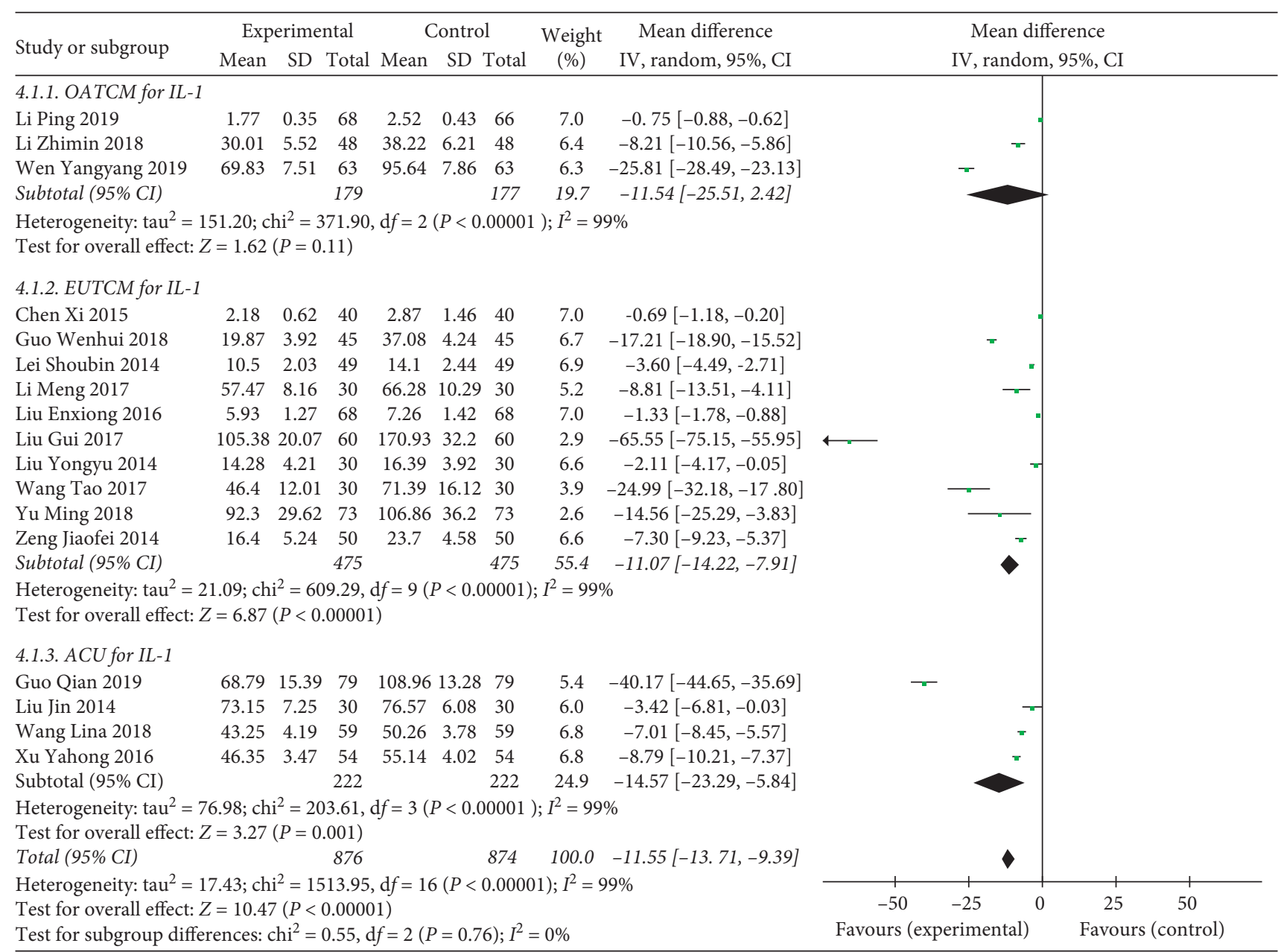

(a)

Figure 6: Continued. 


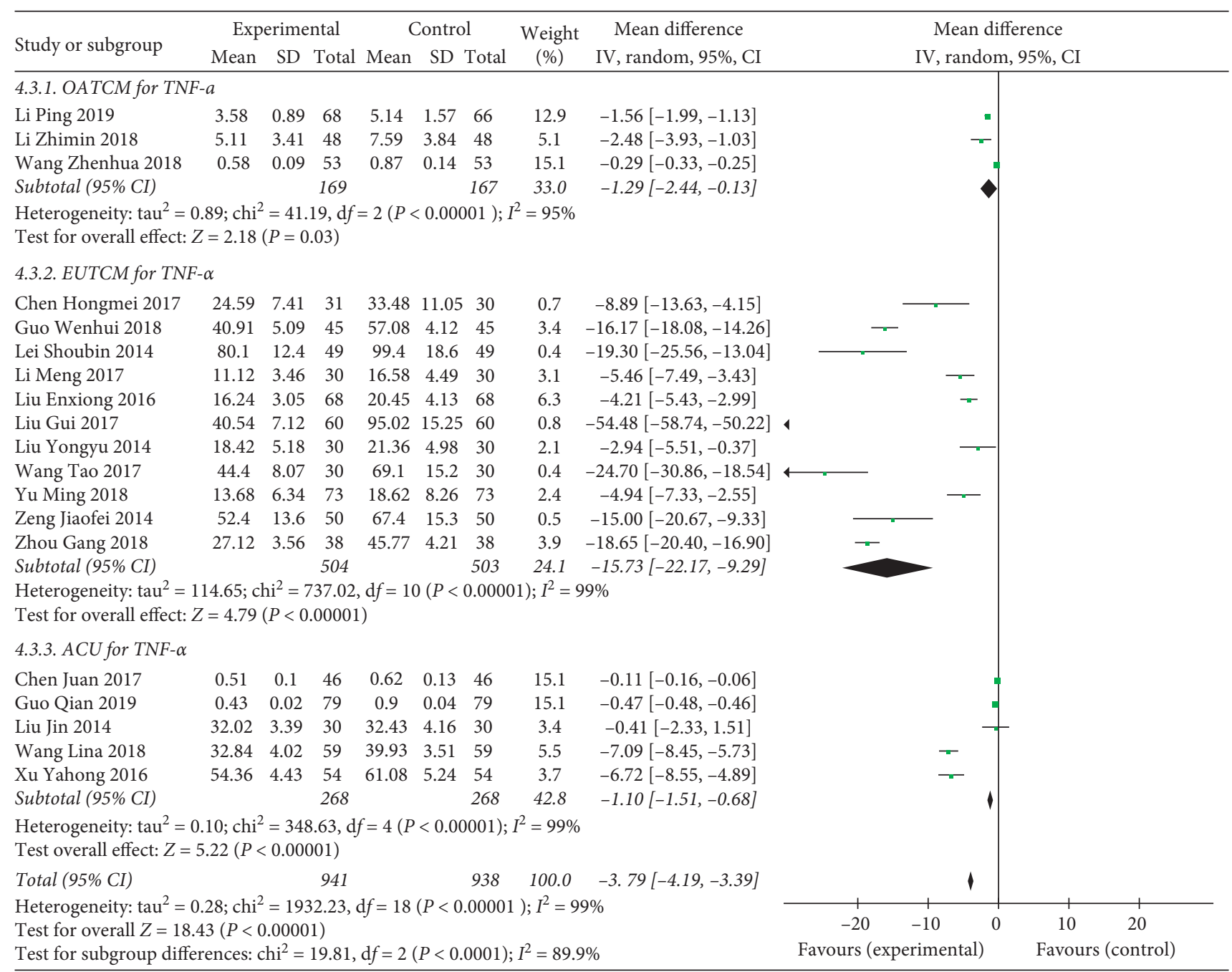

(b)

Figure 6: Continued. 


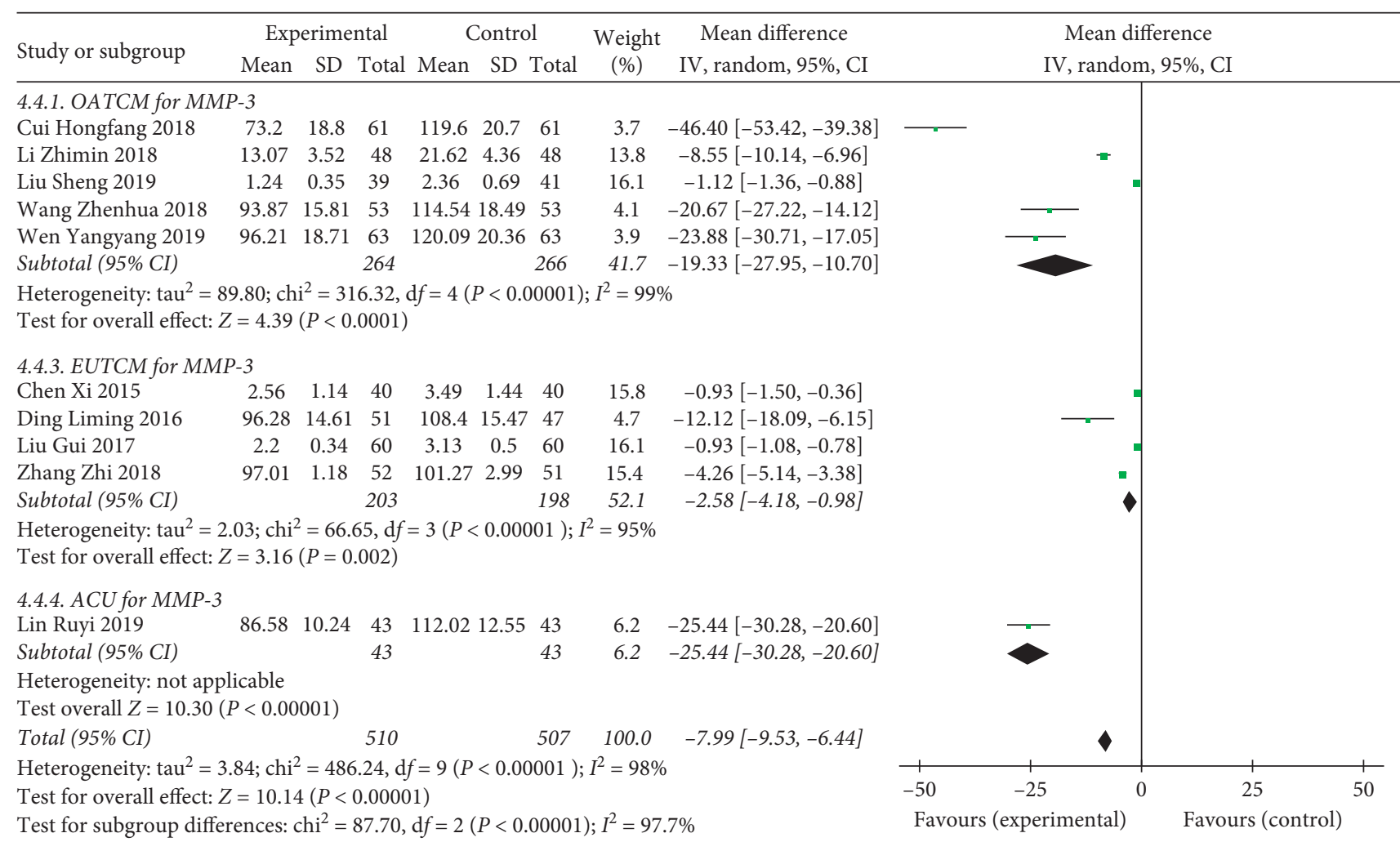

(c)

FIgURE 6: Forest plot of inflammatory cytokines in patients treated with TCM therapy and Western medicine therapy. (a) The plot of IL-1, (b) the plot of TNF- $\alpha$, and (c) the plot of MMP-3. (I) ${ }^{2}$ and (P) are the criteria for the heterogeneity test. $\bullet$ : pooled mean difference, - $-\mathbf{m}$ : mean difference, and 95\%CI. In the detection of inflammatory factors, due to differences in treatment methods, treatment cycles, detection environments, detection instruments, and detection personnel, the detection values vary greatly, but the directions are the same. Therefore, this indicator can be evaluated by the evaluation manager 5.3 .

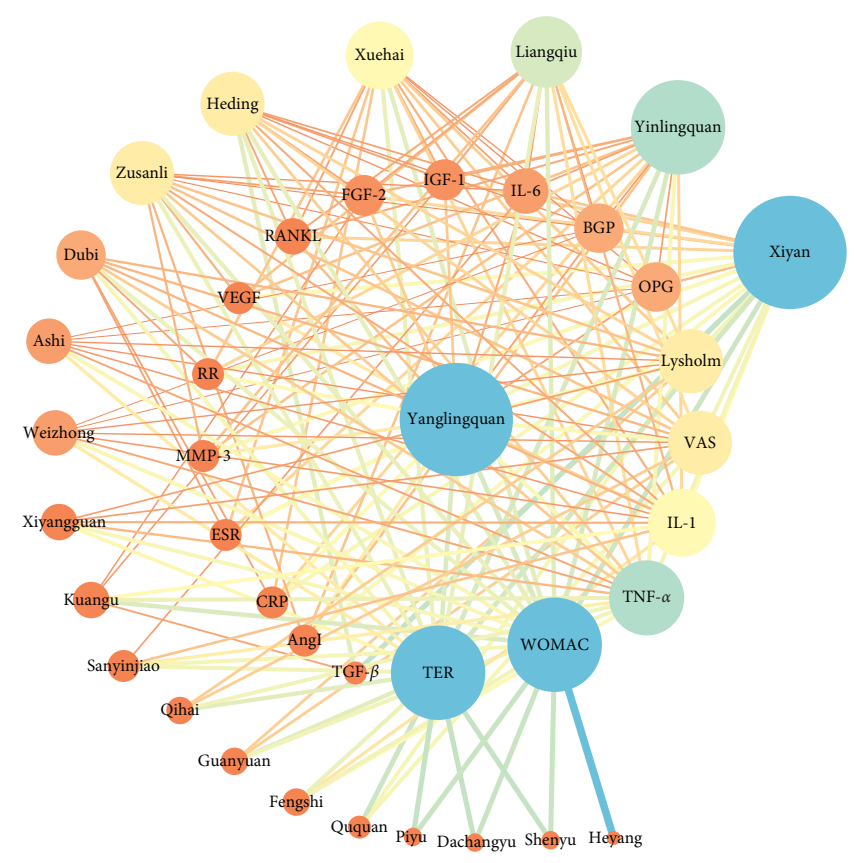

(a)

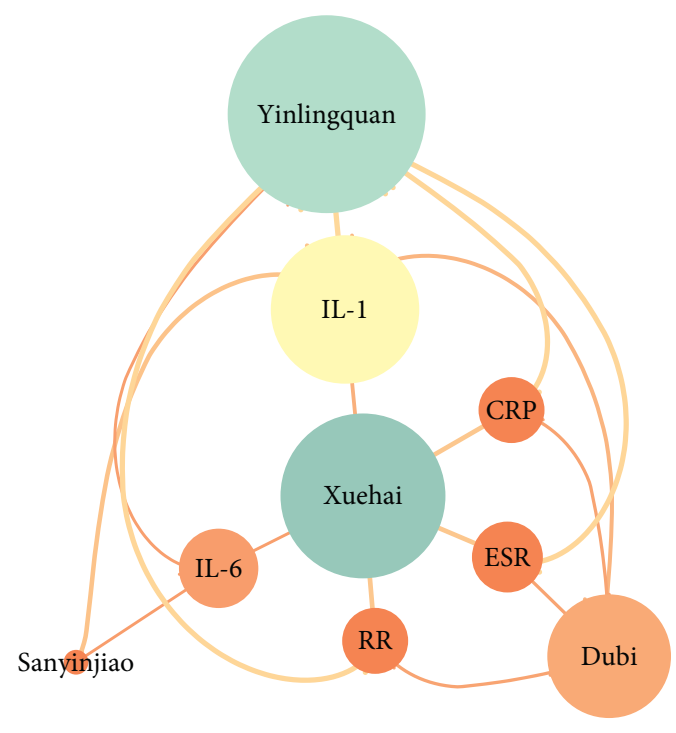

(b)

Figure 7: Continued. 


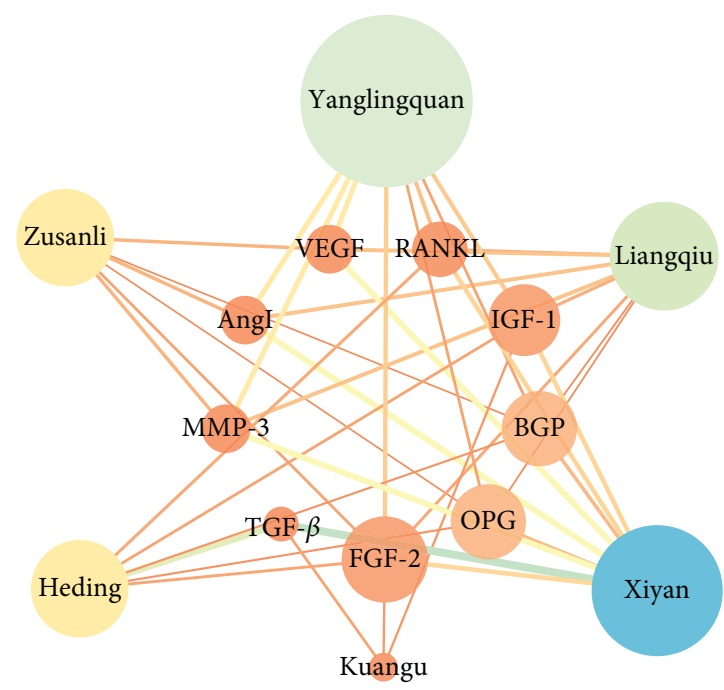

(c)

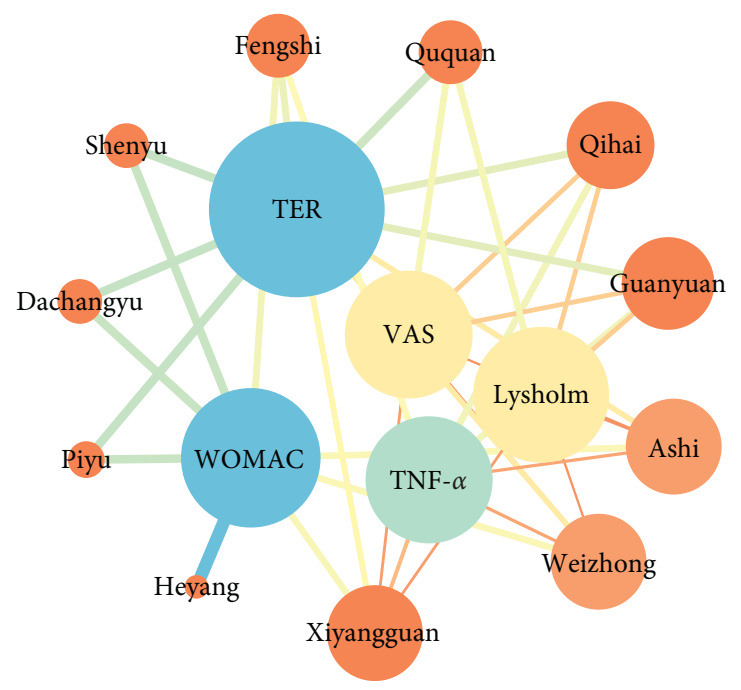

(d)

FIgURE 7: Corresponding relationship between the acupoint and index network. The larger nodes are represented by the main correspondence.

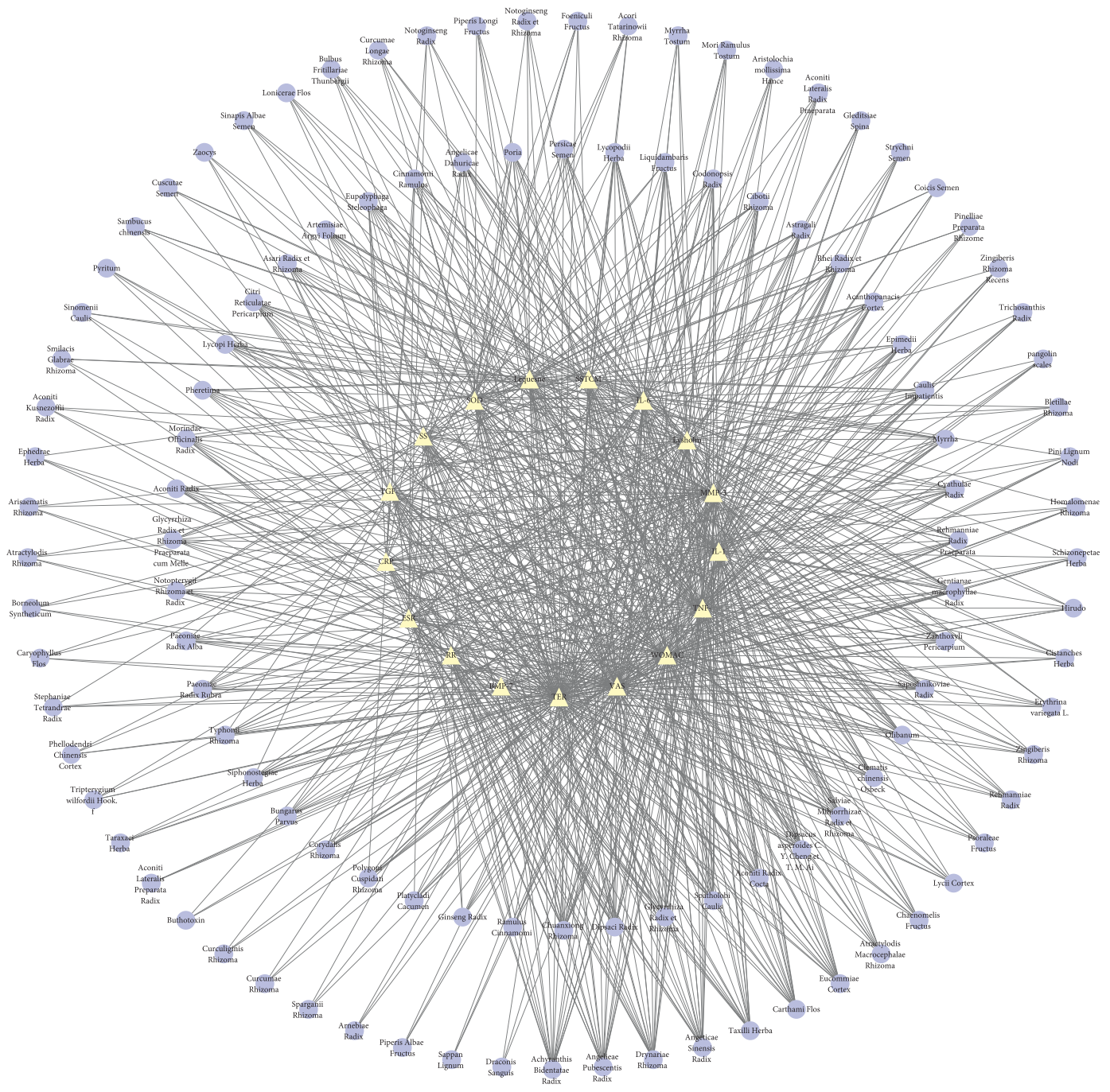

(a)

Figure 8: Continued. 


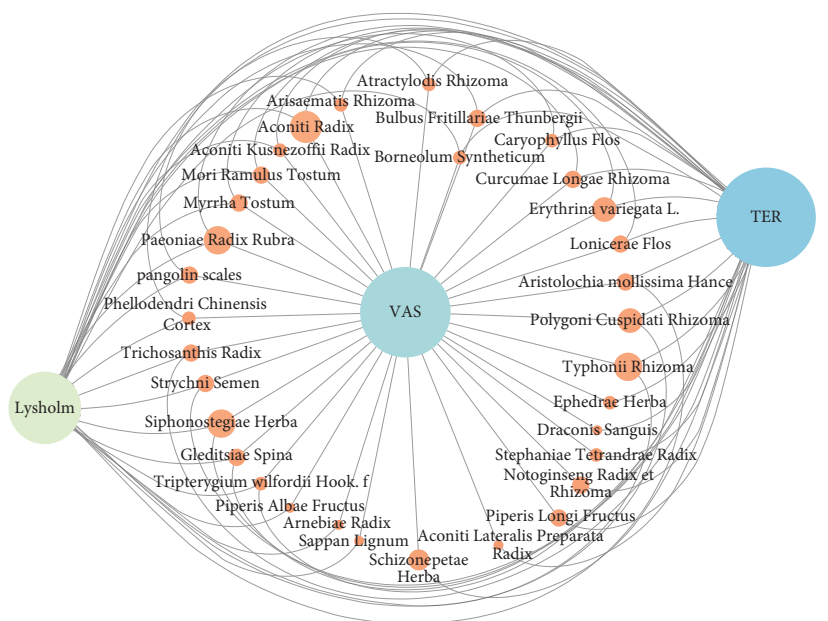

(b)

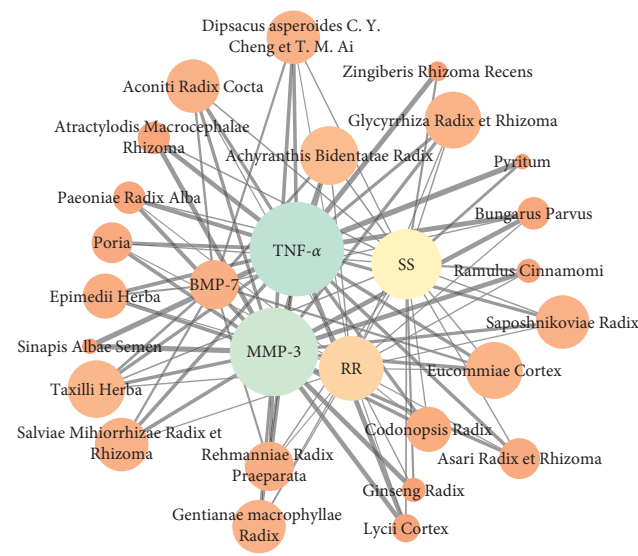

(d)

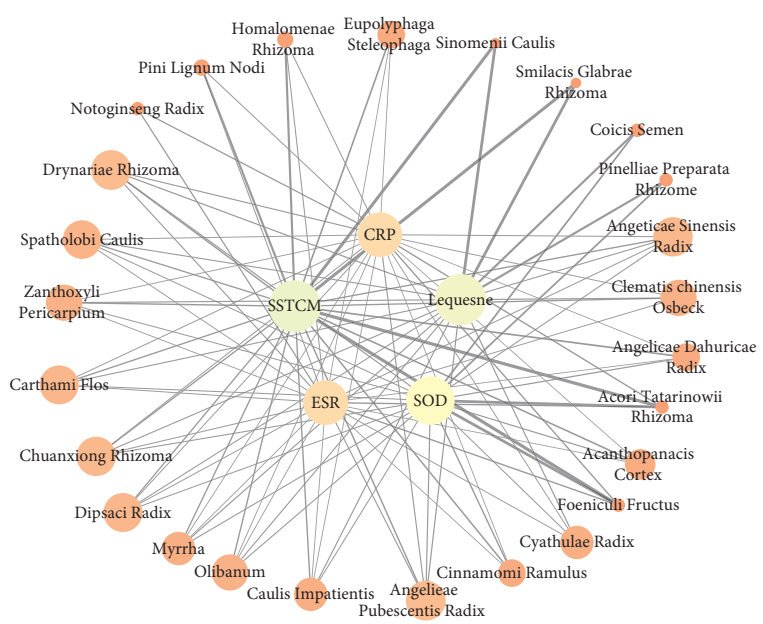

(c)

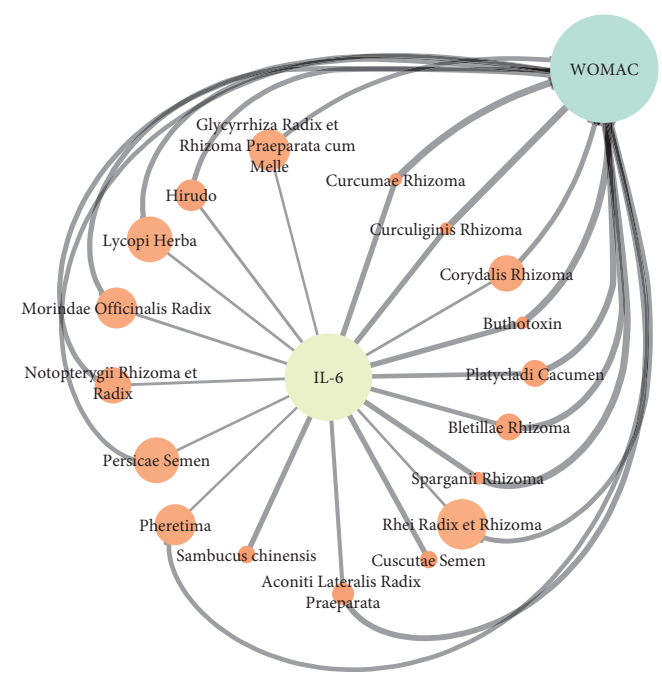

(e)

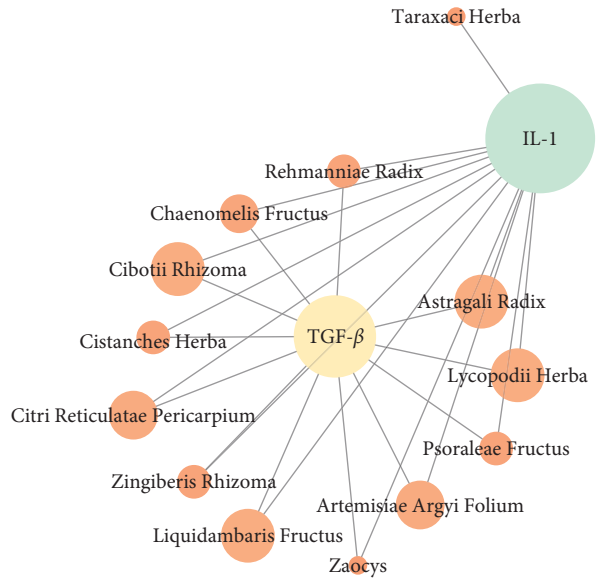

(f)

FIGURE 8: Corresponding relationship between TCMs and the index network. The larger nodes are represented by the main correspondence.

effectively reduce the content of IL- 6 and then reduce the WOMAC. Figure 8(f) shows that Lycopodii Herba, Astragali Radix, and Liquidambaris Fructus significantly reduce the content of IL-1 in OA, indicating that these TCMs have a significant effect on the treatment of patients with OA (Figure 8). 

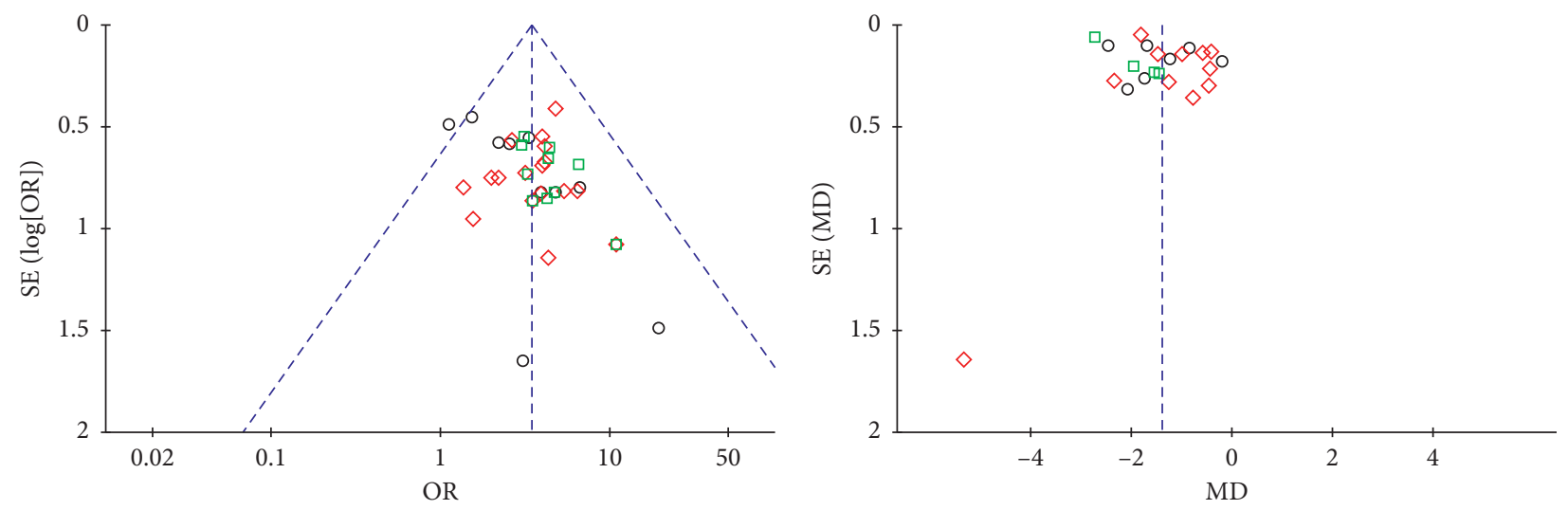

\section{Subgroups \\ - OATCM for OA \\ $\diamond$ EUTCM for OA \\ $\square$ Ac for TER}

(a)

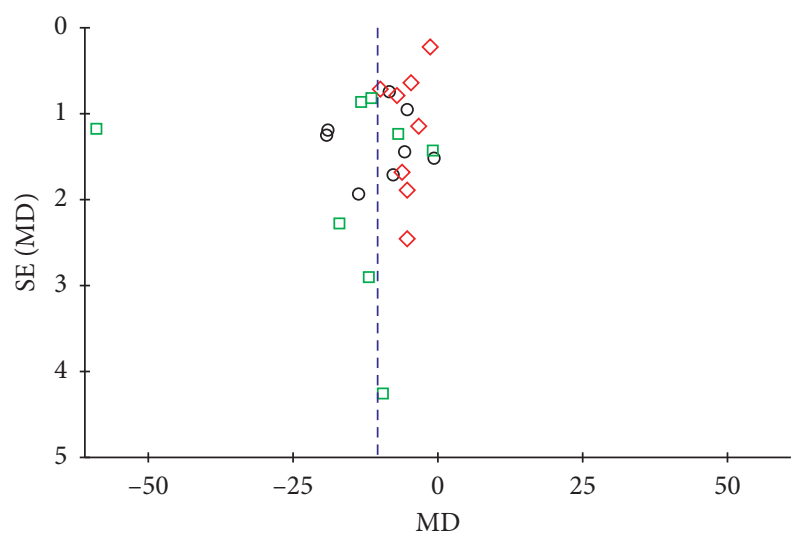

Subgroups

O OATCM for WOMAC

$\diamond$ EUTCM for WOMAC

$\square$ Ac for WOMAC

(c)
Subgroups
O OATCM for VAS
$\diamond$ EUTCM for VAS
$\square$ Ac for VAS

(b)

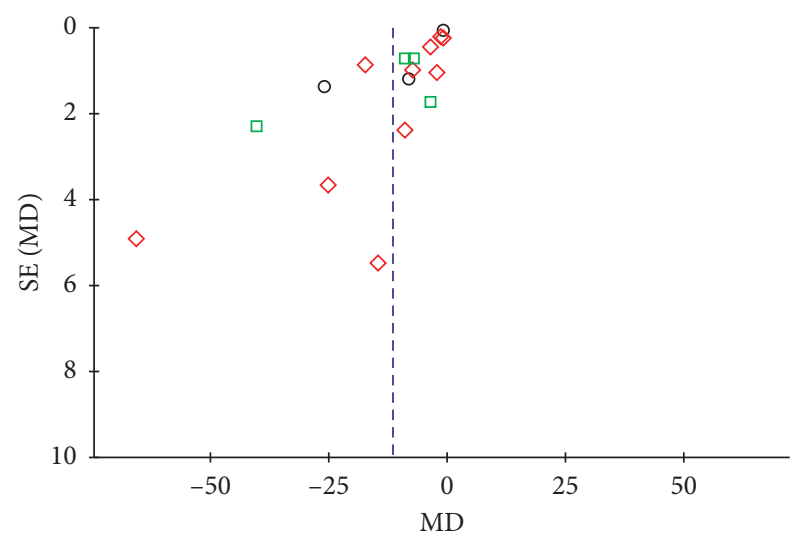

$\diamond$ OATCM for IL-1
$\diamond$ EUTCM for IL-1
$\square$ Ac for IL-1

(d)

FIGURE 9: Funnel plot for the publication bias. (a) The plot of TER, (b) the plot of VAS, (c) the plot of WOMAC, and (d) the plot of IL-1.

3.6. Publication Bias. Publication bias was expressed by a funnel plot. In this study, funnel plots of TCM therapy vs. Western medicine therapy on TER, VAS, WOMAC, and IL1 were applied. The plot is basically symmetrical except that there is some bias due to different usage methods of TCMs in the EUTCM group, which indicates that there is no obvious publication bias (Figures 9(a)-9(d)).

\section{Discussion}

$\mathrm{OA}$ is a degenerative disease that causes pain, stiffness, and decline in body function [6]. With the combined effects of ageing and increasing obesity in the global population, along with increasing numbers of joint injuries, the occurrence of diseases such as OA is becoming more prevalent, with worldwide estimates suggesting that 250 million people are currently affected [58]. At this stage, there are great risks for the various diagnosis and treatment effects of Western medicine, and there is a lack of high-quality data on the benefits and hazards of adverse drug reactions [59]. Current treatments for OA are inadequate [60]. In the theory of TCM, OA belongs to the category of osteoarthralgia, which has been recorded in various literature. The Chinese medicine is through the classification of the causes of OA, symptomatic administration, and the use of appropriate methods of treatment [34]. In the treatment of OA, the use of TCM has few side effects and remarkable curative effects. In contemporary development, the treatment of TCM will be more and more popular among the public.

The results of this meta-analysis show that the treatment of TCM has a significant TER for OA and could reduce the RR. Moreover, there were significant differences in WOMAC $\quad(P<0.00001), \quad$ VAS $\quad(P<0.00001), \quad$ IL-1 $(P<0.00001)$, TNF- $\alpha(P<0.00001)$, BGP $(P=0.007)$, and 
other indicator factors in patients with OA treated by TCM. It could regulate the expression of inflammatory factors, improve the synthesis and invasion of reactive oxygen species on chondrocytes collagen, reduce cartilage damage, block bone destruction, balance bone absorption and bone metabolism, reduce pain in patients, and promote the body's self-activity function [61-64].

ACU is an effective physiotherapy for the treatment of OA with few side effects [65]. The compatibility of multiple acupoints focuses on dispelling wind and activating collaterals, eliminating arthralgia, and relieving pain [66]. Combined with the usual treatment of OA and the acupoints summed up by the network pharmacological map, it can be concluded that Yinlingquan (ST9), Xiyan (EXLE4), Weizhong (BL40), Xuehai (SP10), Yanglingquan (GB34), and Zusanli (ST36) have a great effect on the treatment of OA. According to the analysis of the relationship between TCMs and the index network, Aconiti Radix, Achyranthis Bidentatae Radix, Typhonii Rhizoma, Persicae Semen, and Chuanxiong Rhizoma are very effective in the treatment of OA. The use of these TCMs and acupoints can not only significantly reduce inflammatory factors but also reduce pain and improve the quality of life of patients.

After a comprehensive analysis of the included literature, it was found that there were some limitations: 1. Although the 44 included studies were all RCTs, most of them did not describe the allocation scheme and allocation concealment in detail, which may lead to selection bias and implementation bias. 2. In this included experiment, all the studies were in Chinese, which may make the data collection incomplete and, thus, may have a biased effect on the analysis results. 3. The literature included in this paper lacks uniformity in reference standards for total efficiency and does not strictly distinguish their differences, which may affect the reliability of the results. Therefore, it is suggested that the criteria of clinical efficacy should be unified in future studies. 4. There are deviations in the quality and objectivity of the literature in the selection of research. In addition, the clinical risk assessment and report of the included literature are not clear, which may have a certain impact on the demonstration strength of TCM efficacy. 5. The indicators included in this meta-analysis have some heterogeneity, which may have an impact on the accuracy and objectivity of the results. Therefore, in the future systematic review and meta-analysis, more large samples and high-quality RCTs should be included. It is also hoped that the later RCTs can clarify the specific methods of randomized trials, and more rigorous clinical trials can be conducted to improve the reliability of the clinical effects of TCM.

Compared with other articles, this meta-analysis adopted a more systematic literature retrieval method and included a large sample size. It also contained more comprehensive outcome measures, making this meta-analysis more complete and reliable. With the help of the network relationship construction model in network pharmacology, network analysis was conducted on all TCMs, acupoints, and corresponding indicators involved in this meta-analysis, so as to find the most core relationship between TCM diagnosis and treatment of OA.

\section{Conclusions}

The use of various treatments of TCM can significantly improve the level of TER and reduce the occurrence of RR in the later stage of treatment. These effects are mediated by a combination of several mechanisms. This method of treatment with TCM can reduce pain, improve their ability to move, and improve their living standards by reducing the level of VAS, WOMAC, SSTCM, SS, and Lequesne and increasing the level of Lysholm. TCM therapy can reduce inflammation and exert anti-inflammatory effects by reducing the levels of IL-1, IL-6, TNF- $\alpha$, MMP-3, ESR, and CRP and increasing TGF- $\beta$. TCM therapy can increase BGP, FGF-2, IGF-1, and OPG and reduce BMP-7 and RANKL levels to improve bone metabolism in order to achieve the balance of bone metabolism. The TCM therapy can also reduce the levels of VEGF and Ang I, reduce cartilage injury, and restore vascular endothelial function. The effect of TCM therapy can also increase the level of SOD and reduce the existence of reactive oxygen species. The net analysis of ACU and index showed that ACU at local acupoints such as Yinlingxue, Xuehai, and Yanglingquan can reduce inflammatory indexes, reduce cartilage damage, balance bone metabolism, reduce WOMAC, and improve TER. The network analysis chart of TCMs and index showed that TCMs can effectively reduce WOMAC through the inhibition of IL-1 and MMP-3 and improve the effect of TER and so on. However, our findings must be handled with care because of the small size and low quality of the clinical trial samples cited. Other rigorous and large-scale RCTs are needed to confirm these results.

\section{Data Availability}

The data used to support the findings of this study are included within the supplementary information files and the article

\section{Conflicts of Interest}

The authors declare that they have no conflicts of interest.

\section{Authors' Contributions}

All authors contributed to the study conception and design. Material preparation and data collection were performed by Lin Wang, Xiao-fei Zhang, and Xue zhang. Yu-wei Duan and Zhi-chao Wang analyzed the data. The first draft of the manuscript was written by Lin Wang. All authors commented on previous versions of the manuscript and amended the paper. All authors read and approved the final manuscript. Lin Wang, Xiao-fei Zhang, and Xue zhang contributed equally to this work. 


\section{Acknowledgments}

This work was supported by Chinese Medicine Pharmaceutical Key Discipline of Shaanxi Province (grant no. 303061107) and Key Laboratory of Modern Chinese Medicine Preparation (grant no. 2017003). This work was also supported by the Discipline Innovation Team Project of Shaanxi University of Chinese Medicine (2019YL11).

\section{Supplementary Materials}

ESR and CRP are indicators of inflammatory activity in the body; Figure S1 contains the forest plot of ESR and CRP with TCM therapy and Western medicine therapy; Figure S1-A is the plot of ESR, and Figure S1-B is the plot of CRP. Table S1: the prescriptions of TCMs involved in the OATCM and EUTCM; Table S2: acupoints involved in the treatment of OA by ACU; Table S3: international coding corresponding to acupoints; Table S4: TCM therapy vs. Western medicine therapy on self-activity score; Table S5:TCM therapy vs. Western medicine therapy on inflammatory cytokines; Table S6: the level of bone metabolism indexes of TCM therapy vs. Western medicine therapy; Table S7:ACU treatment of TCM therapy vs. Western medicine therapy on vascular function factors; and Table S8: TCM therapy vs. Western medicine therapy on RR and SOD. (Supplementary Materials)

\section{References}

[1] D. Gregori, G. Giacovelli, C. Minto et al., "Association of pharmacological treatments with long-term pain control in patients with knee osteoarthritis: a systematic review and meta-analysis," JAMA, vol. 320, pp. 2564-2579, 2018.

[2] J. W. Bijilsma, F. Berenbaum, and F. P. Lafeber, "Osteoarthritis: an update with relevance for clinical practice," Lancet, vol. 377, pp. 2115-2126, 2011.

[3] T. E. McAlindon, K. Kwoh, E. M. Roos et al., "OARSI guidelines for the non-surgical management of knee osteoarthritis," Osteoarthritis and Cartilage, vol. 22, pp. 363-388, 2014.

[4] O. H. Jeon, C. Kim, S. Rathod et al., "Local clearance of senescent cells attenuates the development of post-traumatic osteoarthritis and creates a pro-regenerative environment," Nature Medicine, vol. 23, pp. 775-781, 2017.

[5] D. C. Bruno, N. Keller, S. Trelle et al., "Effectiveness of nonsteroidal anti-inflammatory drugs for the treatment of pain in knee and hip osteoarthritis: a network meta-analysis," Lancet, vol. 390, pp. e21-e33, 2017.

[6] A. Kirkley, J. Robert Giffin, C. J. Wong et al., "A randomized trial of arthroscopic surgery for osteoarthritis of the knee," The New England Journal of Medicine, vol. 359, pp. 1097-1107, 2008.

[7] K. L. Bennell, T. Egerton, J. Martin et al., "Effect of physical therapy on pain and function in patients with hip osteoarthritis: a randomized clinical trial," JAMA, vol. 311, pp. 1987-1997, 2014.

[8] L. E. Bayliss, D. J. Beard, A. P. Monk et al., "The effect of patient age at intervention on risk of implant revision after total replacement of the hip or knee: a population-based cohort study," Lancet (London, England), vol. 389, pp. 1424-1430, 2017.

[9] C. Zeng, D. Maureen, M. R. LaRochelle et al., "Association of tramadol with all-cause mortality among patients with osteoarthritis," JAMA, vol. 321, pp. 969-982, 2019.

[10] N. E. Lane, D. L. Shelton, C. A. Birbara et al., "Tanezumab for the treatment of pain from osteoarthritis of the knee," The New England Journal of Medicine, vol. 363, pp. 1521-1531, 2010.

[11] B. Chen, C. Mei, X. Lin et al., "Traditional Chinese medications for knee osteoarthritis pain: a meta-analysis of randomized controlled trials," The American Journal of Chinese Medicine, vol. 44, pp. 677-703, 2016.

[12] L-L. Lin, J-F. Tu, J-K. Shao et al., "Acupuncture of different treatment frequency in knee osteoarthritis: a protocol for a pilot randomized clinical trial," Trials, vol. 20, p. 423, 2019.

[13] Z. Jun-Bo, J. Tai, Y. Wang et al., "Therapeutic efficacy of kangfuxin liquid combined with PPIs in gastric ulcer," Evidence-Based Complementary and Alternative Medicine: eCAM 2019, vol. 201913 pages, 2019.

[14] L. Zhimin and L. Jianqiang, "Clinical evaluation of yishen qubi decoction in treating knee osteoarthritis due to deficiency of liver and kidney and its effect on inflammatory factors in joint fluid," Chinese Journal of Basic Medicine in Traditional Chinese Medicine, vol. 24, pp. 627-630, 2018.

[15] Z. Zhi and W. Yehui, "Clinical observation on 52 cases of knee Osteoarthritis treated by Internal Heat Acupuncture combined with fumigation of traditional Chinese Medicine," Jiangsu Journal of Traditional Chinese Medicine, vol. 50, pp. 67-69, 2018.

[16] W. Zhenhua, D. Sineng, R. Jinwei, and L. Shaofan, "Fuyuan tongbi decoction combined with ozone injection on acute degenerative osteoarthritis of lumbar spine in effect, VAS score, ODI index and serum cytokines," Journal of Emergency in Traditional Chinese Medicine, vol. 27, pp. 266-269, 2018.

[17] W. Yuan, "Clinical observation on 30 cases of knee osteoarthritis treated with oral administration and external application of Chinese Medicine," Chinese Journal of Basic Medicine in Traditional Chinese Medicine, vol. 24, pp. 641643, 2018.

[18] L. Yongyu, "Clinical analysis of intra-articular injection of sodium hyaluronate combined with external application of shangbai ointment in the treatment of knee osteoarthritis," Journal of Guangzhou University of Traditional Chinese Medicine, vol. 31, pp. 11-14+19, 2014.

[19] K. Yao, L. Zhengfei, S. Peng, F. Weijun, and C. Shaoqing, "Effect of duhuo jisheng tang combined with acupuncture in treatment of wind cold dampness type knee osteoarthritis," Chinese Journal of Experimental Traditional Medical Formulae, vol. 24, pp. 147-152, 2018.

[20] W. Yangyang, S. Hanrui, J. Yudong, and L. Youwen, "Clinical efficacy of bushen huoxue decoction combined with traditional Chinese medicine treating knee osteoarthritis and its effect on articular cartilage," Chinese Archives of Traditional Chinese Medicine, vol. 37, pp. 100-103, 2019.

[21] X. Yahong, W. Xiaoli, W. Sheping, and Z. Xingman, "Treatment of 54 cases of knee osteoarthritis with warm acupuncture combined with ultrashort wave," Global Traditional Chinese Medicine, vol. 9, pp. 878-880, 2016.

[22] G. Wenhui, "Effect of ozone combined with traditional Chinese medicine ironing therapy on acute attack of senile knee osteoarthritis and its effect on inflammatory factors in joint fluid," Modern Journal of Integrated Traditional Chinese and Western Medicine, vol. 27, pp. 1787-1790, 2018. 
[23] W. Tao, G. Wang, J. Wang et al., "Clinical observation on fumigating Chinese herbal medicine combined with western medicine in treating knee osteoarthritis due to cold and damp block," Chinese Journal of Osteoporosis, vol. 23, pp. 506-510, 2017.

[24] C. Shuping, L. Guo, and X. Xiaogang, "Clinical observation on the treatment of knee osteoarthritis with TCM fumigation and washing therapy combining Qizhu Zhanjin san," Rheumatism and Arthritis, vol. 5, pp. 37-40, 2016.

[25] L. Shoubin, W. Gang, Z. Bin, L. Chungen, and D. Peidong, "Lontophoresis of Chinese medicine combined with sodium hyaluronate injected into articular cavity in the treatment of early stage knee osteoarthritis," Chinese Journal of Experimental Traditional Medical Formulae, vol. 20, pp. 188-191, 2014.

[26] L. Sheng, L. Ling, and F. Wei, "Effect of duhuo xuduan tang for oral administration and iontophoresis on SDF-1/CXCR4 signaling pathway and relevant factors of cartilage degradation in knee joint fluid of patients with knee osteoarthritis with liver and kidney deficiency," Chinese Journal of Experimental Traditional Medical Formulae, vol. 26, pp. 1-7, 2019.

[27] L. Ruyi, S. Dingjiong, Y. Rui, and L. Heping, "Study on significance of MMP-3 level of OPG after warming acupuncture and moxibustion in treatment of knee osteoarthritis," Chinese Archives of Traditional Chinese Medicine, vol. 37, pp. 1485-1487, 2019.

[28] G. Qian, D. Chaohui, and G. Wei, "Treatment of 79 cases of knee osteoarthritis by warming acupuncture and moxibustion at outer qi point," Chinese Journal of Traditional Medical Traumatology \& Orthopedics, vol. 27, pp. 64-66, 2019.

[29] L. Ping, "Clinical efficacy of modified zhenwu tang combined with zhengji technique on cold-dampness arthralgia syndrome caused by knee osteoarthritis (KOA) at episode," Chinese Journal of Experimental Traditional Medical Formulae, vol. 25, pp. 1-9, 2019.

[30] Y. Ming, Z. Yanjiu, and Z. Dong, "Clinical effect and mechanism of traditional Chinese medicine ion implantation combined with knee arthroplasty in the treatment of knee osteoarthritis," Medical Journal of West China, vol. 30, pp. 1138-1142, 2018.

[31] L. Meng, "Clinical study of arthroscopic debridement combined with Chinese medicine iontophoresis in the treatment of knee osteoarthritis," Chinese Journal of Traditional Medical Traumatology \& Orthopedics, vol. 25, no. 25-28, p. 32, 2017.

[32] W. Lina, W. Sufang, and W. Xiaoning, "Clinical Analysis of 59 cases of knee Osteoarthritis treated by warming Acupuncture combined with dispelling Wind and removing dampness, eliminating arthralgia and relieving pain," Journal of Guangxi Medical University, vol. 35, pp. 1692-1695, 2018.

[33] L. Lin, "Clinical research of professor Ding E in treating knee osteoarthritis by cold paralysis with traditional Chinese medicine fumigation," Chinese Journal of Clinical Healthcare, vol. 21, pp. 555-559, 2018.

[34] H. Junlei, Z. Renzhuo, C. Chaolu, and W. Yueqi, "Clinical observation of juanbi decoction combined with joint mobilization on treatment of knee osteoarthritis," Chinese Journal of Experimental Traditional Medical Formulae, vol. 22, pp. 168-171, 2016.

[35] L. Jin, "Observation of curative effect on osteoarthritis by bushen-huoxue decoction combined with acupuncture and effect on the expression of inflammatory in joint fluid," Chinese Journal of Traditional Medical Traumatology \& Orthopedics, vol. 22, pp. 9-11, 2014.
[36] Z. Jiaofei, "Xianfang huoming decoction jiawei iontophoresis analysis combined with clinical of arthroscopic debridement for osteoarthritis of knee," Chinese Journal of Experimental Traditional Medical Formulae, vol. 20, pp. 199-202, 2014.

[37] L. Jianwu, S-Y. Xiang, Y-B. Feng et al., "Clinical observation on cake-separated mild-warmmoxibustion for treatment of knee osteoarthritis," Chinese Acupuncture \& Moxibustion, vol. 28, pp. 17-19, 2008.

[38] C. Hongmei, Y. Yongping, and G. Qin, “The clinical effect of traditional Chinese medicine fumigation combined with extracorporeal shock wave therapy on TNF- $\alpha$ and IL- 6 of patients with knee osteoarthritis," Journal of Emergency in Traditional Chinese Medicine, vol. 26, pp. 1996-1998, 2017.

[39] C. Hongfang, "Clinical observation of Bushen Huoxue recipe combined with diclofenac sodium sustained release tablets in the treatment of knee osteoarthritis with kidney deficiency and blood stasis," Hebei Journal of Traditional Chinese Medicine, vol. 40, pp. 246-249, 2018.

[40] L. Gui, "Clinical observation on treatment of knee osteoarthritis (wind-cold arthralgia syndrome) with damp-hot compress of traditional Chinese medicine for dispelling cold and soothing tendons combined with glucosamine sulfate," Journal of Emergency in Traditional Chinese Medicine, vol. 26, pp. 1484-1486, 2017.

[41] Z. Gang, "Clinical observation on 38 cases of liver-kidneydeficiency knee osteoarthritis treated by washing and external application of shujin jiegu tang," Rheumatism and Arthritis, vol. 7, pp. 13-16, 2018.

[42] L. Falan, Y. Meihua, L. Yongshan, C. Jiajia, and Z. Jieqiong, "Effect of acupuncture on bone metabolism of articular fluid in patients with knee osteoarthritis," Journal of Yunnan University of Traditional Chinese Medicine, vol. 41, pp. 73-75, 2018.

[43] L. Enxiong, W. Weimin, W. Changguo, and W. Zhao, "External Application of traditional Chinese Medicine combined with glucosamine Hydrochloride capsule in the treatment of 68 cases of senile knee Osteoarthritis," Global Traditional Chinese Medicine, vol. 9, pp. 1357-1359, 2016.

[44] X. Chen, G. Yanan, and Y. Huasheng, "Observations on the efficacy of needle warming therapy plus auricular point sticking and medication in treating knee osteoarthritis," Shanghai Journal of Acupuncture and Moxibustion, vol. 37, pp. 1286-1290, 2018.

[45] W. Xiaoling, W. Xiangbin, H. Meijin, W. Huahua, and J. Feng, "Warm-needling moxibustion for knee osteoarthritis:a randomized controlled trial," Chinese Acupuncture \& Moxibustion, vol. 37, pp. 457-462, 2017.

[46] C. Xi, H-Y. N., Z. Xuemin, and S. Lifang, "Clinical study on Jianpi Bushen Tongluo Zhitong therapy combined with traditional Chinese medicine iontophoresis in treating knee osteoarthritis," China Journal of Traditional Chinese Medicine and Pharmacy, vol. 30, pp. 943-946, 2015.

[47] Z. Tao, X. Zhiguo, X. Zhiyu, and Z. Guojiang, "Clinical effect of bushen huoxue prescription on knee osteoarthritis," Chinese Archives of Traditional Chinese Medicine, vol. 37, pp. 1506-1509, 2019.

[48] L. Linzhong, "Clinical study on the treatment of knee osteoarthritis through the combination of prescription of reinforcing kidney and activating blood and arthroscopic surgery," Medical Innovation of China, vol. 11, pp. 49-52, 2014.

[49] D. Liming and L. Deshan, "Efficacy of arthroscopic debridement combined with fumigation and washing of traditional Chinese medicine in the treatment of knee 
osteoarthritis," Chinese Journal of Gerontology, vol. 36, pp. 2213-2214, 2016.

[50] C. Juan, S. Juan, C. Zheng, and H. Wenhao, "Efficacy of warming acupuncture combined with sodium hyaluronate intraarticular injection in the treatment of osteoarthritis and the influence on MMPs," World Chinese Medicine, vol. 12, pp. 3102-3105, 2017.

[51] Y. Fang, H. Xiaojin, S. Jun, Z. Lin, and Z. Yiqun, "Effect of gubi R ecipe in treating knee osteoarthritis with symptom of kidney deficiency and collateral obstruction," Chinese Journal of Experimental Traditional Medical Formulae, vol. 24, pp. 207-211, 2018.

[52] Z. Yanzhen, L. Cong, Z. Chen, S. Songge, and Q. Xinping, "Evaluation of clinical efficacy and safety of external application of Chinese herbal medicine fumigation with Eliminating Dampness and Dispelling Cold Decoction for knee osteoarthritis syndrome of cold-dampness due to kidney deficiency," China Medical Herald, vol. 15, pp. 105-109, 2018.

[53] D. Jingming, C. Ying, and W. Shengxu, "Comparative study of the efficacies of warm needling versus salt moxibustion in treating knee osteoarthritis," Shanghai Journal of Acupuncture and Moxibustion, vol. 34, pp. 243-245, 2015.

[54] L. Jiangming, Y. Ye, and Z. Hongshen, "The clinical curative effect observation of warm acupuncture combined with acupuncture treatment of knee osteoarthritis by phlebotomy," China \& Foreign Medical Treatment, vol. 35, pp. 171-173, 2016.

[55] Z. Hui, B. Chunqiang, Y. Dawei, S. Xue, and W. Jianmin, "Clinical observation of traditional Chinese medicine leg balneotherapy in treating knee osteoarthritis," Chinese Journal of Experimental Traditional Medical Formulae, vol. 22, pp. 176-180, 2016.

[56] L. Chao, C. Jiaming, and X. Bin, "Clinical study of needle warming moxibustion combined with a new knee moxibustion box in the treatment of knee osteoarthritis," China Journal of Traditional Chinese Medicine and Pharmacy, vol. 31, pp. 3344-3347, 2016.

[57] D. Minghui, L. Yan, and Z. Hong, "Warm acupuncturemoxibustion in patients with osteoarthritis of knee," Chinese Journal of Rehabilitation Medicine, vol. 24, pp. 321-324, 2009.

[58] D. J. Hunter and B.-Z. Sita, "Osteoarthritis," Lancet, vol. 393, pp. 1745-1759, 2019.

[59] E. E. Kerbs, A. Gravely, S. Nugent et al., "Effect of opioid vs nonopioid medications on pain-related function in patients with chronic back pain or hip or knee osteoarthritis pain: the SPACE randomized clinical trial," JAMA, vol. 319, pp. 872882, 2018.

[60] S. P. Messier, G. D. Miller, A. Guermazi et al., "Effects of intensive diet and exercise on knee joint loads, inflammation, and clinical outcomes among overweight and obese adults with knee osteoarthritis: the IDEA randomized clinical trial," JAMA, vol. 310, pp. 1263-1273, 2013.

[61] M. B. Goldring, "The role of cytokines as inflammatory mediators in osteoarthritis: lessons from animal models," Connective Tissue Research, vol. 40, pp. 1-11, 1999.

[62] J. L. Scott, C. Gabrielides, R. Davidson et al., "Superoxide dismutase downregulation in osteoarthritis progression and end-stage disease," Annals of the Rheumatic Diseases, vol. 69, pp. 1502-1510, 2010.

[63] X. Tan, "Comparative analysis of serum proteomes: discovery of proteins associated with osteonecrosis of the femoral head," Translational Research: The Journal of Laboratory and Clinical Medicine, vol. 148, pp. 114-119, 2006.
[64] G. P. Geba, A. L. Weaver, A. B. Polis, M. E. Dixon, and T. J. Schnitzer, "Efficacy of rofecoxib, celecoxib, and acetaminophen in osteoarthritis of the knee: a randomized trial," JAMA, vol. 287, pp. 64-71, 2002.

[65] N. Ding, J. Jiang, Z. Li et al., "Mast cells are important regulator of acupoint sensitization via the secretion of tryptase, 5-hydroxytryptamine, and histamine," PloS One, vol. 13, Article ID e0194022, 2018.

[66] X. Yangkai, F. G. Zhu, C. L. Fan et al., "External therapies of traditional Chinese medicine combined with sodium hyaluronate injected in articular cavity therapy on knee osteoarthritis: meta-analysis," China Journal of Chinese Materia Medica, vol. 43, pp. 1934-1939, 2018. 